\title{
苯并咪唑并氮杂糖的设计、合成及其糖苷酶抑制活性
}

\author{
刘旭苏路路周昭希牛丽萍高利刚琚欢欢 \\ 李丰兴李小六陈华* \\ (河北大学化学与环境科学学院 河北省化学生物学重点实验室 河北保定 071002)
}

\begin{abstract}
摘要 鉴于前期研究发现的源于 $D$-核糖的苯并咪唑并氮杂糖 $\mathbf{1}$ 和 2 具有良好的 $\beta$-葡萄糖糖苷酶抑制活性，通过关键的 Mitsunobu 反应，设计合成了系列新型 $L$-核糖源和 2-脱氧- $D$-核糖源的苯并咪唑并氮杂糖衍生物 $6 \mathbf{a} \sim 6 \mathbf{c}$ 和 7a $\sim 7 \mathbf{c} ;$ 并依 据电子等排的药物设计方法, 设计了系列新的糖环上 2 位氨基取代的稠合三环氮杂糖 $13 \mathrm{a} 、 13 \mathrm{~b}$ 和 $17 \mathrm{a} \sim 17 \mathrm{e}$; 以及 4 位 烷氧基取代的氮杂糖 28a 和 28b. 化合物 13a 和 13b 通过苄胺对甲磺酰化的着基(OMs)取代合成，化合物 17a $\sim 17 \mathrm{e}$ 通过 氨基对三元环氧中间体 15 的开环制备，化合物 $28 \mathrm{a}$ 和 $28 \mathrm{~b}$ 通过 4-羟基对卤代烃的亲核取代合成. 测试了化合物 $6 \mathrm{a} \sim 6 \mathrm{c} 、$ $7 \mathrm{a} \sim 7 \mathrm{c} 、 13 \mathrm{a} 、 13 \mathrm{~b} 、 15 、 17 \mathrm{a} \sim 17 \mathrm{e} 、 19 、 28 \mathrm{a}$ 和 $28 \mathrm{~b}$ 对 $\alpha$-葡萄糖糖苷酶(黑曲霉)、 $\beta$-葡萄糖糖苷酶(杏仁)和 $\alpha$-半乳糖糖 苷酶(咖啡豆)的抑制活性，结果显示所测化合物在 $10 \mu \mathrm{mol} / \mathrm{L}$ 时对 $\alpha$-葡萄糖糖苷酶和 $\alpha$-半乳糖糖苷酶没有或微弱的抑制 活性，部分化合物表现出较好的 $\beta$-葡萄糖糖苷酶抑制活性，其中环氧中间体 15 和 2-氨基化合物 $17 \mathbf{a}$ 活性最好， IC 50 值 分别为 10.5 和 $11.7 \mu \mathrm{mol} / \mathrm{L}$ ，但均低于阳性对照品 $\mathbf{1}$ 的活性. 结果表明该类稠合三环氮杂糖是一类良好的选择性 $\beta$-葡萄 糖苷酶抑制剂.
\end{abstract}

关键词＼cjkstart稠合三环氮杂糖; Mitsunobu 反应; 糖苷酶抑制剂; 苯并咪唑; 环氧中间体

\section{Design and Synthesis of Benzimidazole-Iminosugars and Their Inhibitory Activities against Glycosidases}

\author{
Liu, $\mathrm{Xu} \quad \mathrm{Su}$, Lulu \\ Zhou, Zhaoxi \\ Niu, Liping \\ Gao, Ligang \\ $\mathrm{Ju}$, Huanhuan \\ Li, Fengxing \\ Li, Xiaoliu \\ Chen, Hua* \\ (Key Laboratory of Chemical Biology of Hebei Province, College of Chemistry and Environmental Science, \\ Hebei University, Baoding, Hebei 071002)
}

\begin{abstract}
Based on our previous studies that benzimidazole-fused tricyclic iminosugars 1 and $\mathbf{2}$ derived from $D$-ribose inhibited $\beta$-glucosidase significantly, a series of novel tricyclic iminosugars $\mathbf{6 a} \sim \mathbf{6} \mathbf{c}$ and $7 \mathbf{a} \sim 7 \mathbf{c}$ derived from $L$-ribose and 2-deoxy- $D$-ribose, respectively, were designed and synthesized through the key Mitsunobu reaction. Additionally, based on the drug design method of isosterism, a series of tricyclic iminosugars derivitives 13a, 13b and $\mathbf{1 7} \mathbf{a} \sim \mathbf{1 7} \mathbf{e}$ containing amino group on C-2 position, and 28a and 28b with alkoxyl group on C-4 position on sugar ring were designed. Compounds 13a and 13b were synthesized through substitution reaction of benzylamine and methylsulfonide hydroxy (OMs), while compounds $\mathbf{1 7 a} \sim$ 17e were prepared through ring opened reaction of amine and the three-membered epoxy intermediate 15, compounds 28a and 28b were synthesized through nucleophilic substitution reaction of 4-OH and halohydrocarbon. The inhibitory activities of compounds 6a $\sim 6 \mathbf{c}, 7 \mathbf{a} \sim 7 \mathbf{c}, 13 \mathbf{a}, 13 \mathbf{b}, 15,17 \mathbf{a} \sim 17 \mathrm{e}, 19,28 \mathrm{a}$ and 28b against $\alpha$-glucosidase (Aspergillus niger), $\beta$-glucosidase (almonds) and $\alpha$-galatosidase (coffee beans) were tested. The results showed that the tested compounds exhibited no or weak $\alpha$-glucosidase and $\alpha$-galatosidase inhibitory activities at $10 \mu \mathrm{mol} / \mathrm{L}$. Some compounds exhibited good inhibitory activities against $\beta$-glucosidase. Among them, the epoxy intermediate 15 and compound 17a (2-amino) were the best ones with $\mathrm{IC}_{50}$ values of 10.5 and $11.7 \mu \mathrm{mol} / \mathrm{L}$, respectively, but lower than that of the positive control 1 . The results further suggested that such fused tricyclic iminosugars were the excellent and specific inhibitors against $\beta$-glucosidase.
\end{abstract}

Keywords fused tricyclic iminosugar; Mitsunobu reaction; glycosidase inhibitors; benzimidazole; epoxy intermediate

\footnotetext{
* Corresponding author. E-mail: hua-todd@163.com

Received January 30, 2021; revised February 18, 2021; published online March 25, 2021.

Project supported by the National Natural Science Foundation of China (No. 21772031) and the Natural Science Foundation of Hebei Province (No. B2019201398).

国家自然科学基金(No. 21772031)和河北省自然科学基金(No. B2019201398)资助项目.
} 
糖苷酶作为特异性苷键水解酶, 直接参与细胞表 面或生物活性分子如蛋白质、类脂等的糖基化过程 ${ }^{[1]}$, 其非正常表达常导致多类疾病如糖尿病、肿瘤、病毒性 感染、免疫性疾病等的发生、发展 ${ }^{[2]}$. 设计、合成糖苷 酶抑制剂或底物结构类似物, 是当前新药研发、也是糖 化学生物学的热门研究领域之一 ${ }^{[3-5]}$. 亚氨基糖即氮杂 糖(Iminosugar) ${ }^{[6]}$ 是一类糖环上氧原子被氮取代的糖类 似物, 因其结构与天然单糖相似, 且在生理条件下环上 氮原子易被质子化 ${ }^{[7]}$, 能较好地模拟酶反应过渡态糖基 正离子与酶活性部位作用, 具有高效的糖苷酶抑制活 性 ${ }^{[8-11]}$. 构建亚氨基糖环内氮原子的半椅式平面结构或 $\mathrm{sp}^{2}$ 杂化性 $\left(\mathrm{sp}^{2} \text {-iminosugar }\right)^{[12-13]}$, 将会显著提高化合物的 酶抑制活性及选择性.

基于此, 实验室在前期氮杂糖及稠杂多环的研究基 础上 [14-16]，设计合成了系列稠合三环氮杂糖衍生 物 ${ }^{[17-18]}$, 其中含苯并咪唑结构的衍生物 1 和 2 具有良好 的选择性抑制 $\beta$-葡萄糖苷酶的活性(图 1). 初步的构效 分析提示糖环上羟基均处于 $\beta$ 位时, 如化合物 1 和 2 结 构所示, 有利于化合物酶抑制活性的提高. 为进一步探 讨此类化合物的构效关系, 并尝试获得更高活性的化合 物, 本文设计合成了系列含新型的苯并咪唑并氮杂糖稠 合三环衍生物，并测试了化合物的糖苷酶抑制活性.

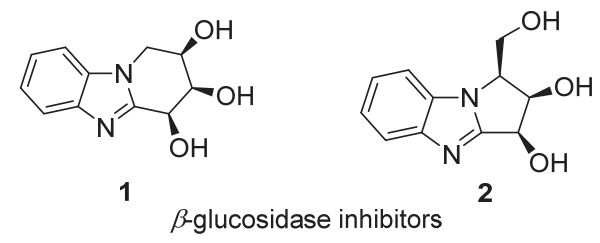

图 $1 \beta$-葡萄糖苷酶抑制剂 1 和 2 的结构

Figure 1 Structures of $\beta$-glucosidase inhibitors 1 and 2

\section{1 结果与讨论}

\section{$1.1 L$-核糖、2-脱氧- $D$-核糖源的苯并咪唑并氮杂糖的 合成}

考虑到化合物 $\mathbf{1}$ 和 $\mathbf{2}$ 是 $D$-核糖源的苯并咪唑并氮杂 糖衍生物, 参照文献[18]方法, 设计合成了其它核糖如 $L$-核糖、2-脱氧- $D$-核糖源的衍生物. 以 $L$-核糖为起始原 料(Scheme 1), 经三步常规糖环保护去保护策略: 首先 合成 $L$-核糖甲苷，再用苄基保护羟基，最后水解相应的 甲苷(产率均在 $90 \%$ 以上)得到茮基保护产物 3a-1(吡喃 型)和 3a-2(呋喃型), 两者比例接近 $1:$ 1. 接着以该混合 物和邻苯二胺为原料, 经碘催化诱导的氧化缩合反应, 以 $68 \%$ 的混合产率得到含开链糖基的苯并咪唑衍生物 4a-1 (35\%)和 4a-2 (33\%). 混合物柱层析分离后, 裸露的 $5-\mathrm{OH}$ 或 4-OH 再分别经关键的 Mitsunobu 反应关环得到 茮基保护的苯并咪唑并氮杂糖三环衍生物 5a-1 (83\%)和 5a-2 (80\%). 最后在强酸性条件下脱除茮基，得到目标 产物 6a-1 (80\%)和 6a-2 (81\%). 按照此方法, 又分别以 4,5-二甲基邻苯二胺和 4,5-二氯邻苯二胺为原料制备了 相应的 $L$-核糖源的苯并咪唑并氮杂糖衍生物 6b-1、

6b-2、6c-1 和 6c-2 (Scheme 1). 同理, 以 2-脱氧- $D$-核糖 为起始原料, 经与上述三种邻苯二胺反应，制备了相应 的稠合三环氮杂糖 7a-1、7a-2、7b-1、7b-2、7c-1 和 7c-2(图 $2)$. 不同的是，该类化合物脱除茮基时，用钯碳(Pd-C)催 化加氢的方法较之用酸的方法效果更好.

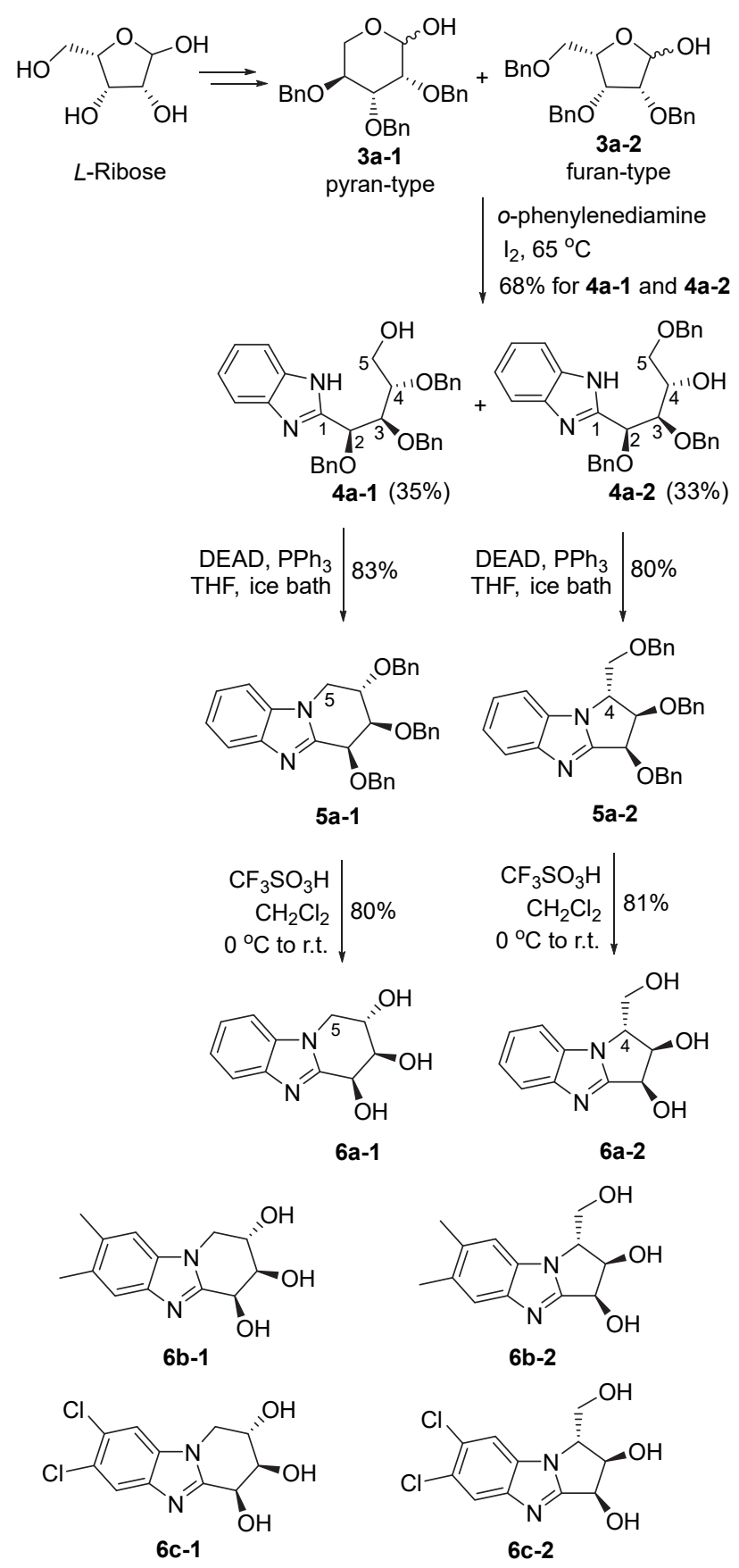

图式 $1 L$-核糖源的苯并咪唑并氮杂糖 $\mathbf{6 a} \sim 6 \mathbf{c}$ 的合成 Scheme 1 Synthesis of benzimidazole-iminosugars $\mathbf{6 a} \sim \mathbf{6 c}$ derived from $L$-ribose 
<smiles>[R20][Y6]O[C@H]1C[C@@H](O)[C@@H](CO)O1</smiles><smiles>Cc1cc2nc3n(c2cc1C)C[C@H](O)[C@H](O)C3</smiles>

$7 b-1$<smiles>OC1Cc2nc3cc(Cl)c(Cl)cc3n2C[C@H]1O</smiles>

7c-1
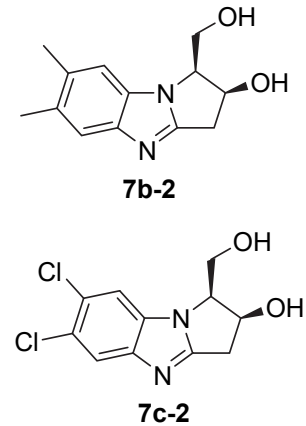

图 22 -脱氧- $D$-核糖源的苯并咪唑并氮杂糖 $7 \mathbf{a} \sim 7 \mathbf{c}$

Figure 2 Benzimidazole-iminosugars $7 \mathbf{a} \sim \mathbf{7} \mathbf{c}$ derived from 2-deoxy- $D$-ribose

化合物的结构、构型可以通过核磁共振 NMR、2D NMR 及高分辨质谱(HRMS)等谱图确证. 通过目标化合 物的碳谱发现, 化合物通过 Mitsunobu 反应关环, 在关 环形成六元环的衍生物中, 其关环碳原子 C-5 的化学位 移要较关环形成五元环的 C-4 在更高场(表 1), 前期研究 中 ${ }^{[18]}$ 也有类似的规律. 因此, 这可以作为此类化合物关 环形成六元环或五元环的判断依据. 此外, 利用化合物 HMBC (Heteronuclear multiple-bond correlation)二维核 磁谱图中 C-1/5-H 或 C-1/4-H 的相关信号(图 3), 也可以 得出相应的关环结论. 如图 3 所示, 化合物 6a-1 和 6c-2 经 ${ }^{1} \mathrm{H}$ NMR, ${ }^{1} \mathrm{H}$ COSY (Correlation spectroscopy) 和 HSQC (Heteronuclear single-quantum correlation)等依次 归属出糖环上所有的 C、 $\mathrm{H}$ 信号, 在相应的 $\mathrm{HMBC}$ 谱中 分别发现 6a-1 中 C-1 ( $\delta 153.8) / 5-\mathrm{H}(\delta 3.83$ 和 $\delta 4.20)$ 以及 6c-2 中 C-1 ( $\delta$ 161.4)/4-H (4.67)的碳氢相关峰, 确证两个 化合物分别是关环形成六元环和五元环的结构, 与表 1 总结出的结果一致. 在 6c-2 的 NOESY (Nuclear overhauser effect spectroscopy)谱中, 没有发现 2-H ( $\delta$ 4.97) 和 4-H 的 NOE 相关峰, 表明 $2-\mathrm{H}$ 与 $4-\mathrm{H}$ 的取向相反, Mitsunobu 关环仍是以构型翻转反应为主, 这也和之前 研究报道一致 ${ }^{[18]}$.

鉴于氨基是羟基的电子等排基团，且含氨基的氮杂 糖也具有良好的选择性糖苷酶抑制活性 ${ }^{[19]}$, 因此以 $D-$ 阿拉伯糖为起始原料, 经上述相同的步骤得到 $D$-阿拉 伯糖源的苯并咪唑并氮杂糖 8 (Scheme 2). 选用 $D$-阿拉 伯糖为起始原料的最初目的是出于后续合成简便, 而且 化合物 8 中 C-2 构型与其它差基取向相反, 有望通过简 单的亲核取代反应实现 $\mathrm{C}-2 \sim \mathrm{C}-4$ 位取代基均处于 $\beta$ 位 置的衍生物. 化合物 8 的 C-3、C-4 位羟基在酸性条件下

表 1 化合物 $6 \mathbf{a} \sim 6 \mathbf{c}$ 和 7a $\sim 7 \mathrm{c}$ 中关环碳的化学位移

Table 1 Chemical shift of the ring closing carbon in compounds $\mathbf{6 a} \sim \mathbf{6} \mathbf{c}$ and $7 \mathbf{a} \sim 7 \mathbf{c}$

\begin{tabular}{cc||cc}
\hline $\begin{array}{c}\mathrm{C}-5 \\
\text { (六元环) }\end{array}$ & $\delta$ & $\begin{array}{c}\mathrm{C}-4 \\
\text { (五元环) }\end{array}$ & $\delta$ \\
\hline $\mathbf{6 a - 1}$ & 44.3 & $\mathbf{6 a - 2}$ & 59.8 \\
$\mathbf{6 b - 1}$ & 44.5 & $\mathbf{6 b - 2}$ & 58.9 \\
$\mathbf{6 c - 1}$ & 44.2 & $\mathbf{6 c - 2}$ & 57.9 \\
$\mathbf{7 a - 1}$ & 45.9 & $\mathbf{7 a - 2}$ & 61.0 \\
$\mathbf{7 b - 1}$ & 45.9 & $\mathbf{7 b - 2}$ & 60.1 \\
$\mathbf{7 c - 1}$ & 45.6 & $\mathbf{7 c - 2}$ & 60.0 \\
\hline
\end{tabular}<smiles>OC1C2C(O)C1N1c3ccccc3NC21</smiles>

6a-1

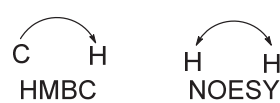

图 3 化合物 6a-1 和 6c-2 中关键的 HMBC 和 NOESY 信号相 关

Figure 3 Key HMBC and NOESY correlations of compounds 6a-1 and 6c-2

用丙叉基保护，得到了化合物 9. 该步反应在室温下进 行, 原料难以反应完全, 产率为 $42 \%$, 升高温度, 副产 物明显增多. 随后化合物 9 中 C-2 位羟基用甲磺酰基 $(\mathrm{Ms})$ 活化, 制备了化合物 10, 同时有少量的(小于 $5 \%$ )氯 代产物 11 生成.
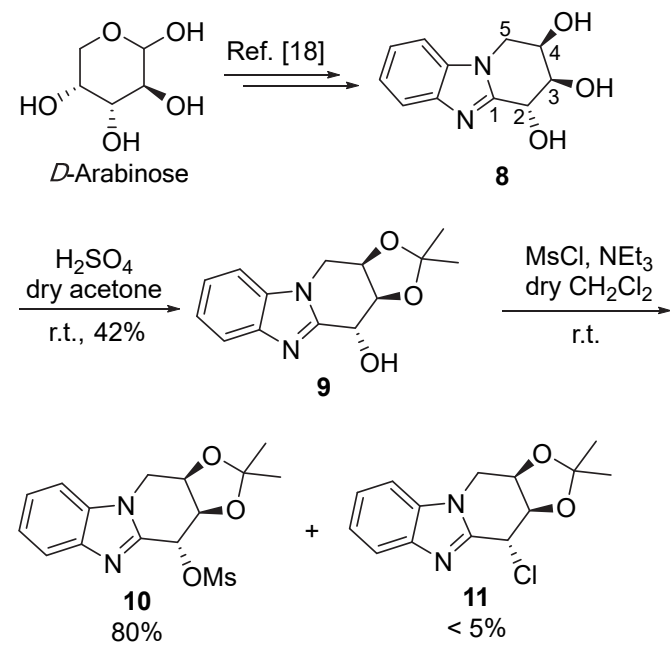

图式 2 化合物 10 和 11 的合成

Scheme 2 Synthesis of compounds $\mathbf{1 0}$ and $\mathbf{1 1}$

\section{$1.2 \mathrm{C}-2$ 位氨基取代的苯并咪唑并氮杂糖衍生物的合} 成

化合物 10 稳定性较差，因此在简单后处理并真空 干燥后，直接用于下一步与茮胺的取代反应，成功得到 
C-2 位芐胺基产物 12a (Scheme 3). 换成 4-氟芐胺为原 料, 也可以得到相应的产物 $\mathbf{1 2 b}$, 但在使用其它脂肪胺 反应时, 反应并不进行, 原料 10 多水解为化合物 $\mathbf{9}$ (意外 的结果, C-2 位羟基构型未翻转). 化合物 $12 \mathrm{a}$ 和 $12 \mathrm{~b}$ 分 别在酸性条件下脱除保护基, 得到化合物 $13 \mathrm{a}$ 和 $13 \mathrm{~b}$ (C-2 位氨基构型也未翻转). 此外, 偶然中发现甲磺酰基 化反应的浓缩液(化合物 10)直接进行红外干燥, 会有丙 叉基脱除且 C-2、C-3 位形成环氧的衍生物 15 生成. 推 测原因可能是在淬灭过量的甲磺酰氯时生成氯化氢, 因 难以洗净, 红外干燥时酸性环境中脱除了丙叉基(化合 物 14). 同时甲磺酰化的羟基(OMs)十分活泼, 继而发生 分子内的亲核反应, 得到了环氧产物 15.
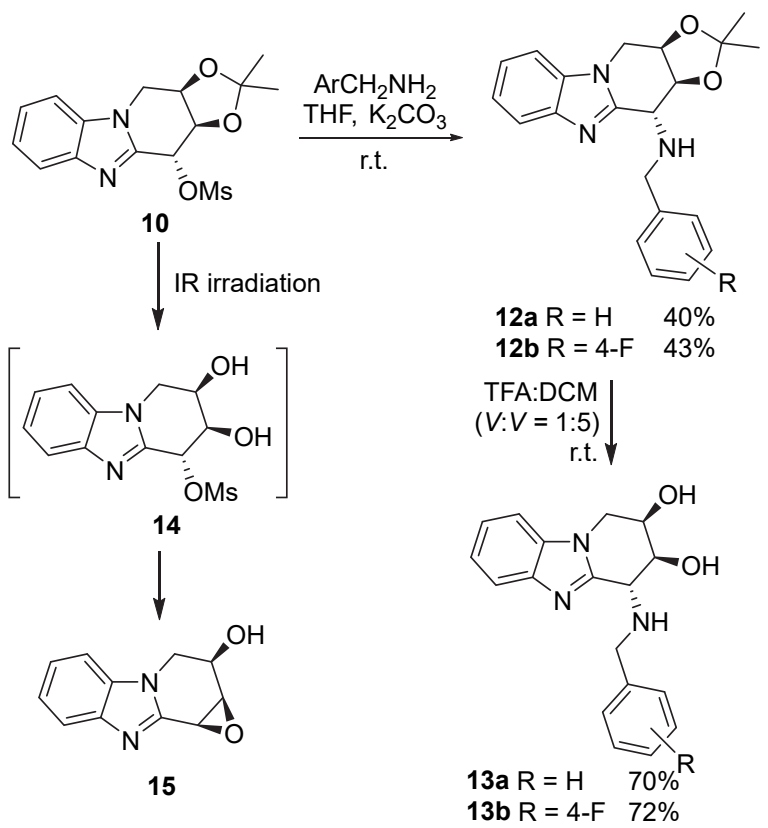

图式 3 化合物 13a 和 $13 b$ 的合成

Scheme 3 Synthesis of compounds 13a and 13b

考虑到三元环氧衍生物是一个活泼中间体，用氨基 取代开环，也可以得到 C-2 位氨基取代衍生物. 因此, 鉴于化合物 11 的稳定可控性, 决定以此为原料, 进行后 续反应. 化合物 11 的大量制备是需要首先解决的问题. 分析化合物 11 是甲磺酰基化过程中有少量的 $\mathrm{HCl}$ 生成, 继而羟基被氯代的结果(Scheme 2). 因此尝试在该步反 应结束后, 向反应液中缓慢滴加与二氯甲烷等体积的水 (1：1), 化合物 11 的收率可提高到 $48 \%$. 继续增加水的 含量, 水解产物 9 增多, 11 的产率下降. 以化合物 11 为 原料，酸性条件下脱除丙叉基得到化合物 16 (Scheme 4). 在室温下 16 会缓慢转化为环氧中间体 $\mathbf{1 5}$, 在强碱 $(\mathrm{NaOH})$ 存在时转化速度加快, 可在 $2 \mathrm{~h}$ 内以 $80 \%$ 的收率 完成. 进一步以氨水或不同的脂肪胺与 15 反应, 顺利得 到预期 C-2 氨基取代衍生物 17a 17e (Scheme 4).

结构上氯代产物 11、茮胺基取代化合物 13a 和 13b

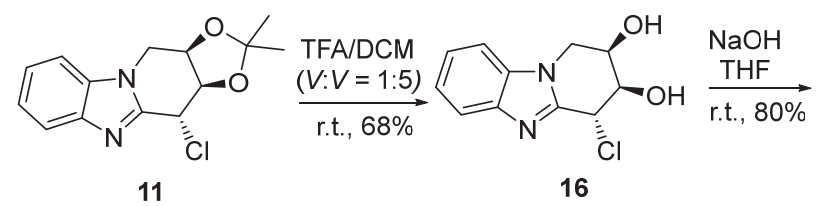<smiles>O[C@H]1Cn2c(nc3ccccc32)[C@@H]2O[C@@H]12</smiles>

15

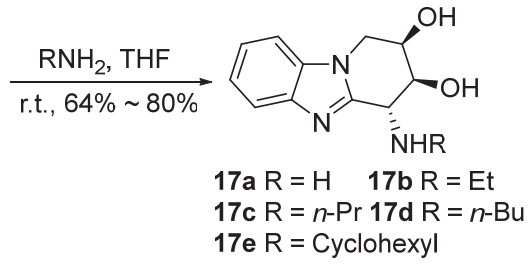

图式 4 化合物 17a $\sim 17 \mathrm{e}$ 的合成

Scheme 4 Synthesis of compounds 17a $\sim 17 \mathrm{e}$

中的 C-2 位均构型保留, 说明取代反应并未发生预期的 构型翻转. 以化合物 13a 的构型分析为例, 通过对其 ${ }^{1} \mathrm{H}-{ }^{1} \mathrm{H}$ COSY 的分析确定 H-2、H-3、H-4 和 H-5 依次归 属于 $\delta 4.08,4.18,4.58,4.33$ 和 4.15. 再经 NOESY 谱图研 究发现(图 4), 2-H 与 4-H 并未发现相关峰, 表明 2-H 与 4-H 的取向相反, C-2 位羟基在苄胺基取代后构型保留. 为了排除苄胺取代基的体积原因，尝试了在甲磺酰基化 反应结束后, 以甲醇直接淬灭 $\mathrm{MsCl}$ (Scheme 5), 得到 C-2 位甲氧基取代产物 18, 脱保护后得到化合物 19. 化 合物 19 经 2D NMR 谱图分析, 仍为 C-2 位构型保留产 物(图 4). 此外, 甲磺酰基化产物 10 经水解也只得到化 合物 9, 说明无论取代基体积大小，在 C-2 位均未发生 构型翻转. 化合物 11 的 X 单晶衍射(图 4)确证氯代亦为 构型保留产物 ${ }^{[20]}$. 造成 C-2 位构型保留的原因, 可能是 由于其邻位 C-3 和 C-4 羟基丙叉基保护后, 形成体积较 大的刚性二氧杂戊环. 大的空间位阻使得取代基难以从 $\mathrm{OMs}$ 基团背面 $\mathrm{S}_{\mathrm{N}} 2$ 亲核进攻 C-2. 由于 $\mathrm{C}-2$ 毗邻 $\mathrm{C}=\mathrm{N}$ 双键，此处发生 $\mathrm{S}_{\mathrm{N}} 1$ 亲核取代的可能性大增，可能的反 应机理如 Scheme 6 所示. 在 OMs 基团离去后，可以形 成比较稳定的烯丙基正离子, 亲核试剂从五元二氧杂环 的背面进攻 C-2 得到相应的产物.

在制备脂肪胺基取代化合物 $17 \mathrm{a} \sim 17 \mathrm{e}$ 时，环氧开 环涉及反应位点问题，即 C-2 位还是 C-3 位被氨基进攻. 理论上, C-2 位酸性较强, 易为开环位点. 以环已胺取代 化合物 17e 为例, 化合物经 ${ }^{1} \mathrm{H}$ NMR, ${ }^{1} \mathrm{H}$ COSY 和 HSQC 依次归属 $\mathrm{C} 、 \mathrm{H}$ 信号，再经 HMBC 谱(图 5), 发现环己基 上的 C-6 ( $\delta$ 54.0) 与糖环上 2-H ( $\delta$ 4.06)的相关峰, 确认 环已氨基在 C-2 位. NOESY 谱图中并未发现 2-H 与 4-H 相关峰, 说明 C-2 位氨基构型保留. 这应该是经由环氧 发生了两次 $\mathrm{S}_{\mathrm{N}} 2$ 亲核取代反应, 得到的产物构型保留.

\subsection{C-4 位烷氧基取代的苯并咪唑并氮杂糖衍生物的 合成}

出于构效分析的目的, 尝试在化合物 1 的 C-4 位引 

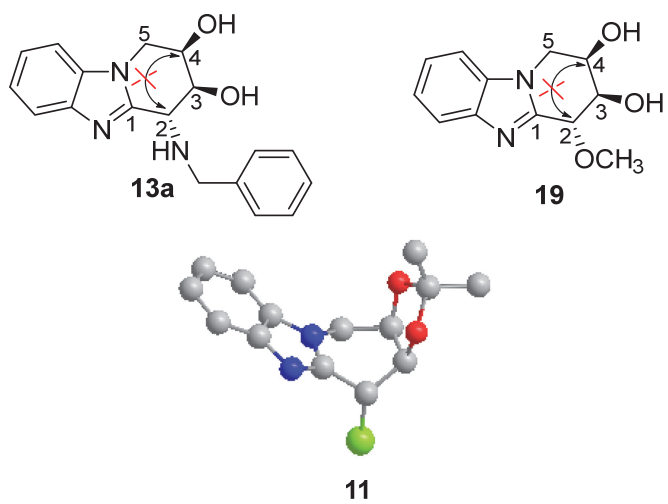

图 4 化合物 $13 a$ 和 19 的 NOESY 信号相关及化合物 $\mathbf{1 1}$ 的 X 单晶衍射结构

Figure 4 NOESY correlations of compounds 13a and 19, and the X-ray crystallographic structure of compound $\mathbf{1 1}$

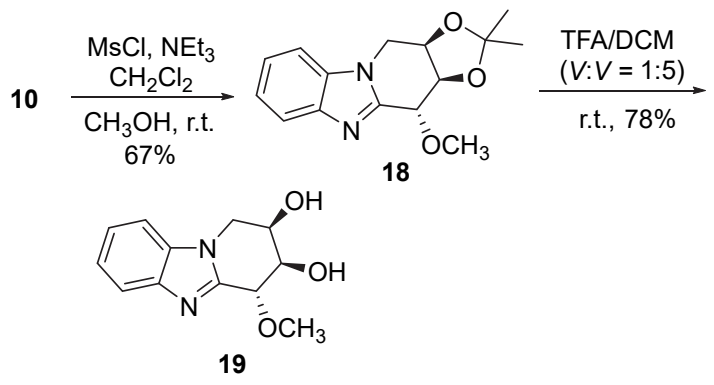

图式 5 化合物 19 的合成

Scheme 5 Synthesis of compound 19

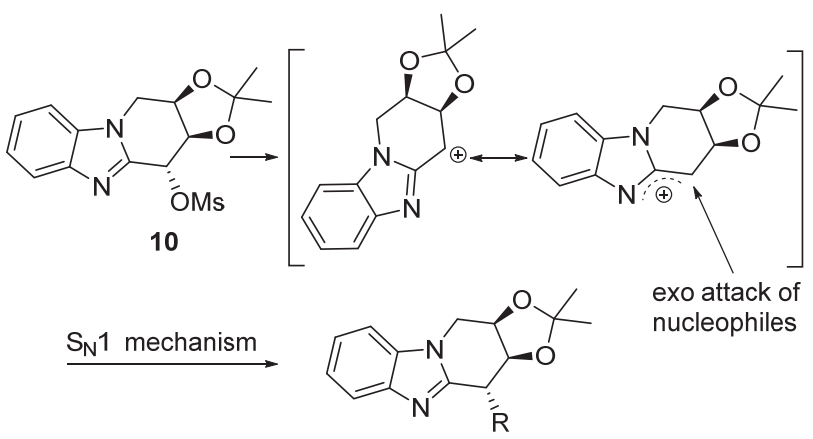

图式 6 C-2 位亲核取代反应的可能机理

Scheme 6 Possible mechanism for the nucleophilic substitution on $\mathrm{C}-2$ position

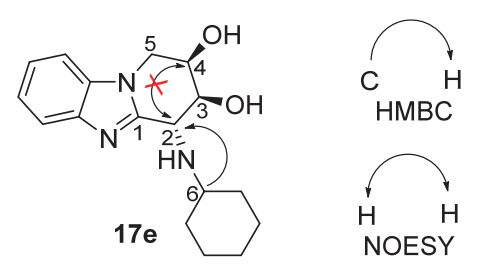

图 5 化合物 $\mathbf{1 7 e}$ 关键的 HMBC 和 NOESY 信号相关

Figure 5 Key HMBC and NOESY correlations of compound $17 \mathrm{e}$
入氨基. 实验以 $L$-核糖源的苯并咪唑并氮杂糖 6a-1 为 原料(Scheme 7), C-2、3 位羟基经丙叉基保护后, C-4 位 着基甲磺酰基化得到化合物 21, 但在碱性(碳酸钠或氢 氧化钠)条件下, 21 与芐胺、脂肪胺等均不反应, 但与化 合物 10 不同的是，化合物 21 非常稳定并且也不发生水 解、醇解. 酸性条件下脱除丙叉基后得到化合物 22 , 但 随后利用获得化合物 15 同样的方法, 并未得到预期的 环氧中间体产物. 以更活泼的三氟甲磺酰基活化 C-4 羟 基得到化合物 23 , 但 23 与苄胺、脂肪胺等也不发生取 代反应，而是生成消去产物 24. 考虑到 C-4 位氨基的取 代反应难以进行，同时烷基化亦能促进氮杂糖生物活性 (如正丁基野尻霉素 ${ }^{[21]}$ ), 进一步设计了 C-4 位含醚氧链 的衍生物. 通过文献方法 ${ }^{[22]}$, 以对甲苯磺酰化(Ts) 的丙 叉基保护的 $D$-核糖和邻苯二胺为原料, 在三氟甲磺酸 钪 $\left[\mathrm{Sc}(\mathrm{OTf})_{3}\right.$ ] 催化下得到化合物 25 (Scheme 8 ). C-4 位羟 基在强碱氢化钠 $(\mathrm{NaH})$ 条件下，与卤代烷烃发生取代反 应得到化合物 $\mathbf{2 7}^{[20]}$, 脱除丙叉基后，得到 C-4 位烷氧基 取代的苯并咪唑并氮杂糖衍生物 28. 此外, 化合物 25 在甲磺酰基化后也不发生氨解、水解等反应. 脱丙叉基 后，可以得到稳定的化合物 26.

\section{4 化合物糖苷酶抑制活性}

以比色法 ${ }^{[18]}$ 测试了所合成化合物 $6 \mathbf{a} \sim 6 \mathbf{6} 、 7 \mathbf{7} \sim 7 \mathbf{c}$ 、 $13 a 、 13 b 、 15 、 17 a \sim 17 \mathrm{e} 、 19 、 28 \mathrm{a}$ 和 $28 b$ 分别对 $\alpha$-葡 萄糖糖苷酶(黑曲霉)、 $\beta$-葡萄糖糖苷酶(杏仁)和 $\alpha$-半乳糖 糖苷酶(咖啡豆)的抑制活性，化合物 $\mathbf{1}$ 为阳性对照. 10 $\mu \mathrm{mol} / \mathrm{L}$ 浓度时抑制率 $50 \%$ 以上的化合物，进一步测试 了其 $\mathrm{IC}_{50}$ 值, 结果见表 2.

由表 2 可知, 所测试化合物 $10 \mu \mathrm{mol} / \mathrm{L}$ 时对 $\alpha$-葡萄 糖糖苷酶(黑曲霉)没有抑制活性，部分化合物对 $\alpha$-半乳 糖糖苷酶(咖啡豆)表现出中等或较弱的酶抑制活性，其 中化合物 $13 \mathrm{~b}$ 的抑制率为 $32.6 \%$. 部分化合物对 $\beta$-葡萄 糖糖苷酶(杏仁)表现出较好的酶抑制活性，其中化合物 15 和 $17 \mathrm{a}$ 在 $10 \mu \mathrm{mol} / \mathrm{L}$ 时的抑制率大于 $50 \%, \mathrm{IC}_{50}$ 值分 别为 10.5 和 $11.7 \mu \mathrm{mol} / \mathrm{L}$, 但均低于阳性对照化合物 $\mathbf{1}$ 的酶抑制活性 $(6.4 \mu \mathrm{mol} / \mathrm{L})$.

以上结果进一步提示苯并咪唑并氮杂糖三环衍生 物是一类良好的选择性的 $\beta$-葡萄糖糖苷酶抑制剂，且糖 环上羟基立体构型变化或 $\mathrm{C}-2$ 位羟基缺失对活性影响较 大, 如 $L$-核糖源和 2-脱氧- $D$-核糖源的衍生物 $6 \mathbf{a} \sim 6 \mathbf{c}$ 和 $7 \mathbf{a} \sim 7 \mathbf{c}$ 均未表现出 $\beta$-葡萄糖糖苷酶抑制活性. 糖环 C-2 位上反式氨基(构型与 $\mathbf{1}$ 中的 2-OH 构型相反)取代(17a) 使化合物 $\beta$-葡萄糖糖苷酶抑制活性略有下降，但较大氨 基(如烷氨基、环已基氨基和苠氨基等)取代则不利于活 性. 糖环 C-4 位上烷氧基也不利于活性. 以上构效关系 提示在此类苯并咪唑类稠合三环氮杂糖糖环上羟基构 


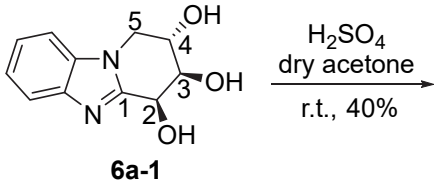

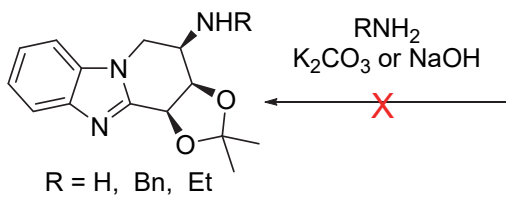<smiles>CO[C@H]1Cn2c(nc3ccccc32)C2OC(C)(C)O[C@H]21</smiles>

21 TFA:DCM
$(V: V=1: 5)$ r.t., $76 \%$<smiles>CN(O)C(C)(C)C</smiles><smiles>CC1(C)OC2c3nc4ccccc4n3C[C@@H](O)C2O1</smiles>

$\mathrm{Tf}_{2} \mathrm{O}$, pyridine $\underset{-20^{\circ} \mathrm{C}, 84 \%}{\mathrm{dry} \mathrm{CH}_{2} \mathrm{Cl}_{2}}$<smiles>CCC1(C)OC2c3nc4ccccc4n3C[C@H](OC)C2O1</smiles>

23

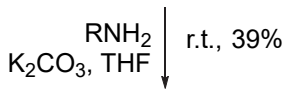<smiles>CC1(C)OC2C=Cn3c(nc4ccccc43)C2O1</smiles>

图式 7 甲磺酰化产物 22 的合成

Scheme 7 Synthesis of mesylated compound 22

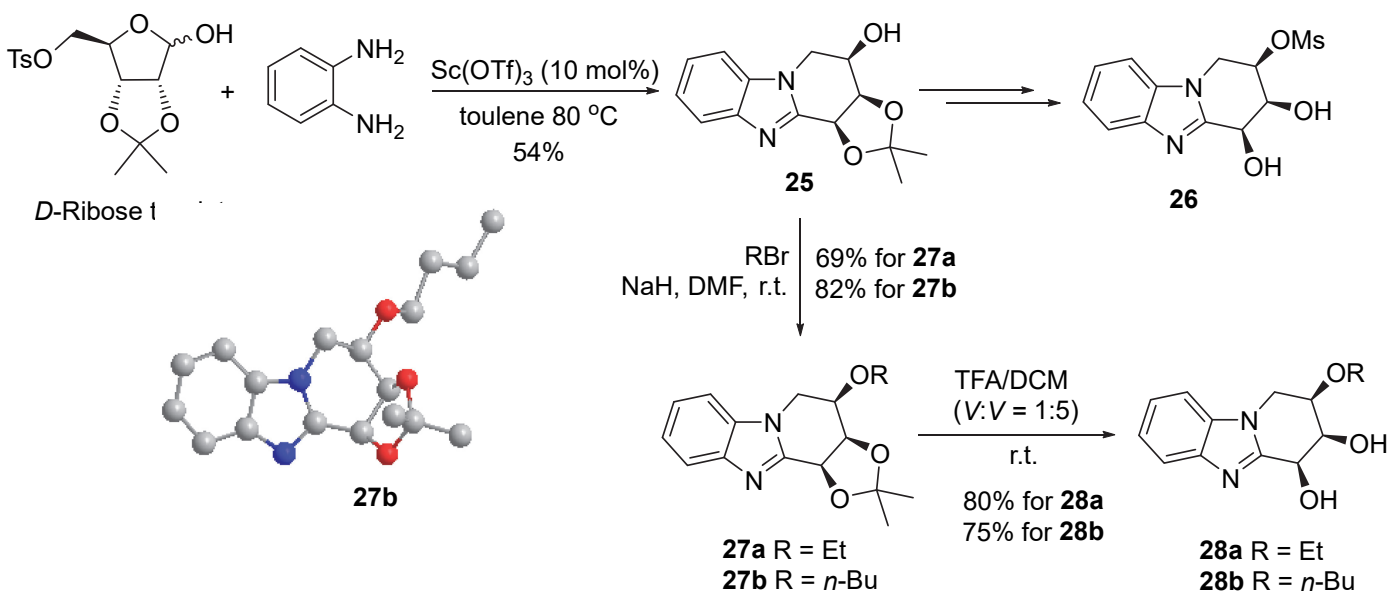

图式 8 C-4 位烷氧基的衍生物 $\mathbf{2 8 a}$ 和 $\mathbf{2 8 b}$ 的合成

Scheme 8 Synthesis of derivatives 28 and $\mathbf{2 8 b}$ with alkoxyl group on C-4 position

型比较重要, 而烷基化结构改造对活性可能不利, 这为 后续化合物结构设计提供了参考, 进一步的合成及生物 活性研究正在进行之中.

\section{2 结论}

设计合成了系列新型 $L$-核糖源、2-脱氧- $D$-核糖源 以及 $\mathrm{C}-2$ 位含氨基的 $D$-阿拉伯糖源的苯并咪唑并氮杂 糖 6a $\sim 6 \mathrm{c} 、 7 \mathrm{a} \sim 7 \mathrm{c} 、 13 \mathrm{a} 、 13 \mathrm{~b} 、 17 \mathrm{a} \sim 17 \mathrm{e} 、 19 、 28 \mathrm{a}$ 和 $\mathbf{2 8 b}$, 以及环氧中间体 15. 部分化合物在 $10 \mu \mathrm{mol} / \mathrm{L}$ 时表 现出较好的 $\beta$-葡萄糖糖苷酶(杏仁)抑制活性, 其中环氧 中间体 15 和含 2-氨基的化合物 $17 \mathbf{a}$ 是所测化合物中活 性最好的两个衍生物, $\mathrm{IC}_{50}$ 值分别为 10.5 和 $11.7 \mu \mathrm{mol} / \mathrm{L}$.
化合物对其它两种糖苷酶 $\alpha$-葡萄糖糖苷酶(黑曲䨋)和 $\alpha$ 半乳糖糖苷酶(咖啡豆)没有或微弱的抑制活性，进一步 提示该类稠合三环氮杂糖是一类良好的选择性的 $\beta$-葡 萄糖糖苷酶抑制剂.

\section{3 实验部分}

\section{1 仪器与试剂}

熔点由 $\mathrm{SGW}^{\circledR} \mathrm{X}-4$ 显微熔点仪(温度计未校正)测定; 核磁共振谱用 BRUKER AC-P600 (600 MHz)型核磁共 振仪或 Brucker advance II 400 FT-NMR 型核磁共振仪测 定，内标为 TMS; 质谱(ESI)用 Agilent G6300 离子阱液 相质谱联用仪; 高分辨质谱(ESI)用 FTICR-MS (Ionspec 


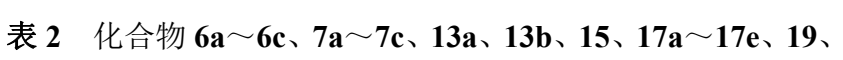
$28 \mathrm{a}$ 和 $28 \mathrm{~b}$ 的糖苷酶抑制活性

Table 2 Inhibitory activities of compounds $\mathbf{6 a} \sim \mathbf{6 c}, 7 \mathbf{a} \sim \mathbf{7 c}$, 13a, 13b, 15, 17a $\sim 17 \mathrm{e}, 19,28 \mathrm{a}$ and $28 \mathrm{~b}$ against glycosidases

\begin{tabular}{|c|c|c|c|}
\hline \multirow[b]{2}{*}{ 化合物 } & \multicolumn{3}{|c|}{ 抑制率/\% } \\
\hline & $\begin{array}{c}\alpha \text {-葡萄糖糖苷酶 } \\
\text { (黑曲霉) }\end{array}$ & $\begin{array}{c}\beta \text {-葡萄糖糖苷酶 } \\
\text { (杏仁) }\end{array}$ & $\begin{array}{c}\alpha \text {-半乳糖糖苷酶 } \\
\text { (咖啡豆) }\end{array}$ \\
\hline $6 a-1$ & $-^{a}$ & - & - \\
\hline $6 a-2$ & - & - & 6.4 \\
\hline $6 b-1$ & - & - & - \\
\hline $6 b-2$ & - & - & - \\
\hline $6 c-1$ & - & - & - \\
\hline $6 c-2$ & 1.2 & 1.6 & - \\
\hline $7 a-1$ & - & 2.0 & - \\
\hline $7 a-2$ & - & - & 8.2 \\
\hline $7 b-1$ & - & - & 5.2 \\
\hline $7 b-2$ & - & - & 2.1 \\
\hline $7 c-1$ & - & 1.4 & - \\
\hline $7 c-2$ & - & - & - \\
\hline $13 a$ & - & - & - \\
\hline $13 b$ & - & - & 32.6 \\
\hline 15 & - & $54.5\left(10.5^{b}\right)$ & 9.8 \\
\hline $17 \mathbf{a}$ & - & $50.4(11.7)$ & 12.2 \\
\hline $17 b$ & - & 3.4 & 8.6 \\
\hline $17 \mathrm{c}$ & - & - & - \\
\hline 17d & - & - & - \\
\hline $17 \mathrm{e}$ & - & - & - \\
\hline 19 & - & 24.1 & - \\
\hline 28a & - & - & - \\
\hline $28 b$ & - & - & - \\
\hline 1 & & $70.6(6.4)$ & \\
\hline
\end{tabular}
bitory concentration $\left(\mathrm{IC}_{50}, \mu \mathrm{mol} / \mathrm{L}\right)$.

7.0T)型质谱仪测定; 旋光为 SGW-1 型自动旋光仪测定, 上海精密科学仪器有限公司; 吸光度在 BioRad 3550 型 酶标仪上测定. $\alpha$-葡萄糖糖苷酶(黑曲霉), $\beta$-葡萄糖糖苷 酶(杏仁), $\alpha$-半乳糖糖干酶(咖啡豆), 以及相应的底物对 硝基苯酚吡喃糖苷均购于 Sigma 公司. 层析用硅胶 (200 300 目)为青岛海洋化工厂产品. 本文所用其他试 剂均为分析纯, 无水试剂均按常规方法处理, 水为二次 蒸馏水.

\section{2 实验方法}

\section{2 .1 化合物 $\mathbf{6 a} \sim \mathbf{6 c}$ 的合成}

称取 3a-1 和 3a-2 的混合物(1.10 g, $2.61 \mathrm{mmol})$ 以及 邻苯二胺( $0.23 \mathrm{~g}, 0.8$ equiv. $)$, 加入 $100 \mathrm{~mL}$ 甲苯溶解, 氮 气保护下 $65{ }^{\circ} \mathrm{C}$ 搅拌反应. 称取碘( $66 \mathrm{mg}, 0.1$ equiv.)用 5 $\mathrm{mL}$ 甲苯溶解, 恒压滴液漏斗滴加到反应瓶中. 反应 24 $\mathrm{h}$ 后用薄层色谱(TLC)检测, 反应完全. 用旋转蒸发仪蒸 除溶剂, 残留物用 $200 \sim 300$ 目硅胶柱层析分离(石油醚/ 乙酸乙酯, $V: V=4: 1)$ 得到产物 4a-1 (0.46 g) 和 4a-2 $(0.43 \mathrm{~g})$ 的混合物.
(2S,3S,4S)-4-(1H-苯并 $[d]$ 咪唑-2-基)-2,3,4-三(苄氧 基)丁烷-1-醇(4a-1)：黄色油状物，产率 35\%. ${ }^{1} \mathrm{H}$ NMR $\left(600 \mathrm{MHz}, \mathrm{CDCl}_{3}\right) \delta: 7.32 \sim 7.23(\mathrm{~m}, 19 \mathrm{H}), 5.22(\mathrm{~d}, J=$ $2.4 \mathrm{~Hz}, 1 \mathrm{H}), 4.93$ (d, $J=10.8 \mathrm{~Hz}, 1 \mathrm{H}), 4.78$ (d, $J=10.8$ $\mathrm{Hz}, 1 \mathrm{H}), 4.62 \sim 4.56(\mathrm{~m}, 3 \mathrm{H}), 4.50(\mathrm{~d}, J=11.4 \mathrm{~Hz}, 1 \mathrm{H})$, $4.29(\mathrm{dd}, J=8.4,2.4 \mathrm{~Hz}, 1 \mathrm{H}), 3.77(\mathrm{dd}, J=12.0,3.6 \mathrm{~Hz}$, $1 \mathrm{H}), 3.72(\mathrm{dd}, J=12.0 \mathrm{~Hz}, 3.0 \mathrm{~Hz}, 1 \mathrm{H}), 3.45 \sim 3.43(\mathrm{~m}$, $1 \mathrm{H}) ;{ }^{13} \mathrm{C}$ NMR $\left(150 \mathrm{MHz}, \mathrm{CDCl}_{3}\right) \delta: 151.7,137.9,137.6$, $137.6,128.6,128.5,128.4,128.3,128.1,128.0,127.8$, 127.8, 80.3, 78.7, 76.9, 75.1, 72.4, 72.1, 60.4; HRMS (ESI) calcd for $\mathrm{C}_{32} \mathrm{H}_{33} \mathrm{~N}_{2} \mathrm{O}_{4}[\mathrm{M}+\mathrm{H}]^{+}$509.2440, found 509.2442 .

( $2 S, 3 R, 4 S)-4-(1 H$-苯并 $[d]$ 咪唑-2-基)-1,3,4-三(苄氧 基)丁烷-2-醇(4a-2): 黄色油状物, 产率 33\%. ${ }^{1} \mathrm{H}$ NMR $\left(600 \mathrm{MHz}, \mathrm{CDCl}_{3}\right) \delta: 7.31 \sim 7.16(\mathrm{~m}, 19 \mathrm{H}), 5.26(\mathrm{~d}, J=$ $2.4 \mathrm{~Hz}, 1 \mathrm{H}), 4.88(\mathrm{~d}, J=11.4 \mathrm{~Hz}, 1 \mathrm{H}), 4.62 \sim 4.54(\mathrm{~m}$, $3 \mathrm{H}), 4.50(\mathrm{~d}, J=12.0 \mathrm{~Hz}, 1 \mathrm{H}), 4.44(\mathrm{~d}, J=12.0 \mathrm{~Hz}, 1 \mathrm{H})$, $4.16(\mathrm{dd}, J=8.4,2.4 \mathrm{~Hz}, 1 \mathrm{H}), 3.64 \sim 3.61(\mathrm{~m}, 1 \mathrm{H}), 3.55(\mathrm{~d}$, $J=4.2 \mathrm{~Hz}, 2 \mathrm{H}) ;{ }^{13} \mathrm{C} \mathrm{NMR}\left(150 \mathrm{MHz}, \mathrm{CDCl}_{3}\right) \delta: 152.0$, $138.1,137.9,137.6,128.5,128.4,128.1,127.9,127.9$, 127.9, 127.8, 127.7, 80.8, 76.9, 74.9, 73.5, 72.2, 70.9, 70.1; HRMS (ESI) calcd for $\mathrm{C}_{32} \mathrm{H}_{33} \mathrm{~N}_{2} \mathrm{O}_{4}[\mathrm{M}+\mathrm{H}]^{+}$509.2440, found 509.2438 .

称取 4a-1 (0.41 g, $0.80 \mathrm{mmol})$ 和三苯基膦 $\left(\mathrm{PPh}_{3} 0.68\right.$ $\mathrm{g}, 3.0$ equiv.), 加入 $20 \mathrm{~mL}$ 无水四氢呋喃(THF)溶解, 冰 浴下摚拌, 氮气保护. 待反应液充分冷却后, 加入偶氮 二甲酸二乙酯(DEAD, $0.45 \mathrm{~g}, 3.0$ equiv.), 撤掉冰浴室温 下反应 $6 \mathrm{~h}$. TLC 检测反应完全, 用旋转蒸发仪蒸除溶 剂, 残留物用 $200 \sim 300$ 目硅胶柱层析分离(石油醚/乙 酸乙酯, $V: V=5: 1$ )得到 $0.33 \mathrm{~g}$ 的产物 5a-1.

$(2 S, 3 R, 4 S)-2,3,4$-三(芐氧基)-1,2,3,4-四氢苯并[4,5]咪唑并 $[1,2-a]$ 吡啶(5a-1): 黄色油状物, 产率 $83 \%$. ${ }^{1} \mathrm{H}$ NMR $\left(600 \mathrm{MHz}, \mathrm{CDCl}_{3}\right) \delta: 7.79 \sim 7.77(\mathrm{~m}, 1 \mathrm{H}), 7.48(\mathrm{~d}$, $J=7.2 \mathrm{~Hz}, 2 \mathrm{H}), 7.37 \sim 7.22(\mathrm{~m}, 16 \mathrm{H}), 5.27(\mathrm{~d}, J=12.0 \mathrm{~Hz}$, $1 \mathrm{H}), 5.01(\mathrm{~d}, J=12.0 \mathrm{~Hz}, 1 \mathrm{H}), 4.92 \sim 4.85(\mathrm{~m}, 2 \mathrm{H}), 4.69$ $(\mathrm{d}, J=3.0 \mathrm{~Hz}, 1 \mathrm{H}), 4.66(\mathrm{~d}, J=11.4 \mathrm{~Hz}, 1 \mathrm{H}), 4.56(\mathrm{~d}, J=$ $12.0 \mathrm{~Hz}, 1 \mathrm{H}), 4.48$ (dd, $J=12.0,7.2 \mathrm{~Hz}, 1 \mathrm{H}), 4.19 \sim 4.17$ $(\mathrm{m}, 2 \mathrm{H}), 3.97(\mathrm{t}, J=7.2 \mathrm{~Hz}, 1 \mathrm{H}) ;{ }^{13} \mathrm{C} \mathrm{NMR}(150 \mathrm{MHz}$, $\left.\mathrm{CDCl}_{3}\right) \delta: 150.5,143.7,138.4,137.4,134.2,128.6,128.4$, $128.2,128.1,128.0,128.0,127.8,127.7,127.6,122.6$, 122.3, 120.0, 109.3, 74.2, 74.0, 73.7, 73.4, 73.0, 71.7, 42.9; HRMS (ESI) calcd for $\mathrm{C}_{32} \mathrm{H}_{31} \mathrm{~N}_{2} \mathrm{O}_{3}[\mathrm{M}+\mathrm{H}]^{+}$491.2334, found 491.2338 .

称取 5a-1 (0.26 g, $0.54 \mathrm{mmol})$ 于 $50 \mathrm{~mL}$ 烧瓶中, 加 入 $12 \mathrm{~mL}$ 无水二氯甲烷 $\left(\mathrm{CH}_{2} \mathrm{Cl}_{2}\right)$ 溶解，冰浴下搅拌，氮 
气保护. 待反应液充分冷却后, 慢慢加入三氟甲磺酸 (0.16 g, 2.0 equiv.), 撤掉冰浴室温下反应 $7 \mathrm{~h}$. TLC 检测 反应完全. 加入固体碳酸钾中和反应液至中性, 用旋转 蒸发仪蒸除溶剂, 残留物用 $200 \sim 300$ 目硅胶柱层析分 离(二氯甲烷/甲醇, $V: V=15: 1$ )得到 $94 \mathrm{mg}$ 的产物 6a-1.

$(2 S, 3 R, 4 S)-1,2,3,4$-四氢苯并 $[4,5]$ 咪唑并 $[1,2-a]$ 吡 啶-2,3,4-三醇(6a-1): 白色固体，产率 80\%. m.p. 201.1 $202.9{ }^{\circ} \mathrm{C} ;[\alpha]_{\mathrm{D}}^{25}-4.0\left(c \quad 0.1, \mathrm{CH}_{3} \mathrm{OH}\right) ;{ }^{1} \mathrm{H}$ NMR $(400$ $\left.\mathrm{MHz}, \mathrm{DMSO}-d_{6}\right) \delta: 7.60 \sim 7.58(\mathrm{~m}, 1 \mathrm{H}), 7.50 \sim 7.48(\mathrm{~m}$, $1 \mathrm{H}), 7.23 \sim 7.17(\mathrm{~m}, 2 \mathrm{H}), 5.75 \sim 7.33(\mathrm{~m}, 3 \mathrm{H}), 4.80(\mathrm{~d}, J=$ $2.4 \mathrm{~Hz}, 1 \mathrm{H}), 4.23 \sim 4.17(\mathrm{~m}, 2 \mathrm{H}), 4.11(\mathrm{~d}, J=1.2 \mathrm{~Hz}, 1 \mathrm{H})$, $3.86 \sim 3.79(\mathrm{~m}, 1 \mathrm{H}) ;{ }^{13} \mathrm{C}$ NMR $\left(100 \mathrm{MHz}, \mathrm{DMSO}-d_{6}\right) \delta$ : $153.8,143.4,134.7,122.3,122.1,119.0,110.5,72.0,66.6$, 66.3, 44.3; HRMS (ESI) calcd for $\mathrm{C}_{11} \mathrm{H}_{12} \mathrm{~N}_{2} \mathrm{O}_{3} \mathrm{Na}[\mathrm{M}+$ $\mathrm{Na}]^{+}$243.0745, found 243.0742.

按照上述相同的方法, 以化合物 4a-2 为原料, 制备 6a-2. 换用 4,5-二甲基邻苯二胺和 4,5-二氯邻苯二胺, 经 相同步骤合成化合物 $6 \mathrm{~b} 1 \sim 6 \mathrm{~b} 2$ 和 $6 \mathrm{c} 1 \sim 6 \mathrm{c} 2$.

$(1 R, 2 R, 3 S)-1$-(着圣甲基)-2,3-二氢- $1 H$-苯并 $[d]$ 吡咯并 [1,2- $a$ ]咪唑-2,3-二醇(6a-2): 白色固体 $75 \mathrm{mg}$, 产率 63\%. m.p. $236.1 \sim 237.8{ }^{\circ} \mathrm{C}$; $[\alpha]_{\mathrm{D}}^{25}-8.0\left(c 0.10, \mathrm{CH}_{3} \mathrm{OH}\right) ;{ }^{1} \mathrm{H}$ NMR $\left(600 \mathrm{MHz}, \mathrm{DMSO}-d_{6}\right) \delta: 7.66 \sim 7.68(\mathrm{~m}, 2 \mathrm{H}), 7.22$ (t, $J=6.6 \mathrm{~Hz}, 2 \mathrm{H}), 5.64$ (t, $J=6.0 \mathrm{~Hz}, 2 \mathrm{H}), 5.21(\mathrm{t}, J=4.8$ $\mathrm{Hz}, 1 \mathrm{H}), 4.88$ (t, $J=6.0 \mathrm{~Hz}, 1 \mathrm{H}), 4.71(\mathrm{t}, J=6.0 \mathrm{~Hz}, 1 \mathrm{H})$, $4.64 \sim 4.61(\mathrm{~m}, 1 \mathrm{H}), 3.97 \sim 3.94(\mathrm{~m}, 1 \mathrm{H}), 3.75(\mathrm{t}, J=6.0$ $\mathrm{Hz}, 1 \mathrm{H}) ;{ }^{13} \mathrm{C}$ NMR (100 MHz, DMSO- $\left.d_{6}\right) \delta: 159.5,146.1$, 132.0, 130.1, 122.8 122.5, 112.7, 73.7, 66.0, 61.3, 59.8; HRMS (ESI) calcd for $\mathrm{C}_{11} \mathrm{H}_{12} \mathrm{~N}_{2} \mathrm{O}_{3} \mathrm{Na}\left[\mathrm{M}+\mathrm{Na}{ }^{+}\right.$ 243.0745, found 243.0748.

$(2 S, 3 R, 4 S)$-7, 8 -二甲基-1,2,3,4-四氢苯并 [4,5]咪唑并 [1,2- $a]$ 吡啶-2,3,4-三醇(6b-1): 白色固体 $104 \mathrm{mg}$, 产率 78\%. m.p. $247.9 \sim 249.3{ }^{\circ} \mathrm{C}$; $[\alpha]_{\mathrm{D}}^{25}-2.0\left(\right.$ c $\left.0.1, \mathrm{CH}_{3} \mathrm{OH}\right)$; ${ }^{1} \mathrm{H}$ NMR (400 MHz, DMSO-d $) \delta: 7.37$ (s, 1H), 7.25 (s, $1 \mathrm{H}), 5.94(\mathrm{~s}, 1 \mathrm{H}), 5.57(\mathrm{~s}, 1 \mathrm{H}), 5.46(\mathrm{~s}, 1 \mathrm{H}), 4.80(\mathrm{~s}, 1 \mathrm{H})$, $4.20(\mathrm{~d}, J=5.6 \mathrm{~Hz}, 1 \mathrm{H}), 4.14 \sim 4.11(\mathrm{~m}, 2 \mathrm{H}), 3.79 \sim 3.75$ $(\mathrm{m}, 1 \mathrm{H}), 2.31(\mathrm{~s}, 3 \mathrm{H}), 2.29(\mathrm{~s}, 3 \mathrm{H}) ;{ }^{13} \mathrm{C} \mathrm{NMR}(100 \mathrm{MHz}$, $\left.\mathrm{CD}_{3} \mathrm{OD}\right) \delta: 160.0,141.1,131.9,131.3,123.7,118.3,109.8$, $71.066 .4,65.9,44.2,19.1,19.0$; HRMS (ESI) calcd for $\mathrm{C}_{13} \mathrm{H}_{16} \mathrm{~N}_{2} \mathrm{O}_{3} \mathrm{Na}[\mathrm{M}+\mathrm{Na}]^{+}$271.1058, found 271.1059.

(1R,2R,3S)-1-(差圣甲基)-6,7-二甲基-2,3-二氢- $1 H$-苯 并 $[d]$ 吡咯并 $[1,2-a]$ 咪唑-2,3-二醇(6b-2): 白色固体 76 $\mathrm{mg}$, 产率 57\%. m.p. 226.7 228.1 ${ }^{\circ} \mathrm{C} ;[\alpha]_{\mathrm{D}}^{25}-13.0$ (c $\left.0.1, \mathrm{CH}_{3} \mathrm{OH}\right) ;{ }^{1} \mathrm{H}$ NMR (400 MHz, $\left.\mathrm{CD}_{3} \mathrm{OD}\right) \delta: 7.56(\mathrm{~s}$, $1 \mathrm{H}), 7.42(\mathrm{~s}, 1 \mathrm{H}), 5.04 \sim 5.03(\mathrm{~m}, 1 \mathrm{H}), 4.95 \sim 4.94(\mathrm{~m}$,
1H), $4.83(\mathrm{~s}, 1 \mathrm{H}), 4.16(\mathrm{~d}, J=12.0 \mathrm{~Hz}, 1 \mathrm{H}), 4.00 \sim 3.96$ (m, 1H), 2.37 (s, 3H), 2.29 (s, 3H); ${ }^{13} \mathrm{C}$ NMR (100 MHz, $\left.\mathrm{CD}_{3} \mathrm{OD}\right) \delta: 157.4,142.4,133.3,132.9,129.3,118.0,111.8$, 72.8, 65.3, 61.0, 57.9, 19.1, 19.0; HRMS (ESI) calcd for $\mathrm{C}_{13} \mathrm{H}_{16} \mathrm{~N}_{2} \mathrm{O}_{3} \mathrm{Na}[\mathrm{M}+\mathrm{Na}]^{+}$271.1058, found 271.1056.

$(2 S, 3 R, 4 S)-7,8$-二氯-1,2,3,4-四氢苯并 [4,5]咪唑并 [1,2- $a$ 吡啶-2,3,4-三醇(6c-1): 白色固体 $115 \mathrm{mg}$, 产率 74\%. m.p. $197.8 \sim 199.3{ }^{\circ} \mathrm{C}$; $[\alpha]_{\mathrm{D}}^{25}-8.0\left(\right.$ c 0.1, $\left.\mathrm{CH}_{3} \mathrm{OH}\right)$; ${ }^{1} \mathrm{H}$ NMR (400 MHz, DMSO- $\left.d_{6}\right) \delta: 7.90(\mathrm{~s}, 1 \mathrm{H}), 7.84$ (s, $1 \mathrm{H}), 5.90(\mathrm{~s}, 1 \mathrm{H}), 5.55(\mathrm{~s}, 1 \mathrm{H}), 5.42(\mathrm{~s}, 1 \mathrm{H}), 4.82(\mathrm{~s}, 1 \mathrm{H})$, $4.25 \sim 4.22(\mathrm{~m}, 2 \mathrm{H}), 4.10(\mathrm{~s}, 1 \mathrm{H}), 3.80(\mathrm{t}, J=12.4 \mathrm{~Hz}, 1 \mathrm{H})$; ${ }^{13} \mathrm{C}$ NMR (100 MHz, DMSO- $\left.d_{6}\right) \delta: 156.7,143.0,134.3$, 124.7, 124.6, 120.2, 112.6, 71.9, 66.6, 66.0, 44.5; HRMS (ESI) calcd for $\mathrm{C}_{11} \mathrm{H}_{10} \mathrm{Cl}_{2} \mathrm{~N}_{2} \mathrm{O}_{3} \mathrm{Na}[\mathrm{M}+\mathrm{Na}]^{+}$310.9966, found 310.9961 .

$(1 R, 2 R, 3 S)-6,7$-二氯-1-(羟甲基)-2,3-二氢- $1 H$-苯并 $[d]$ 吡咯并 $[1,2-a]$ 咪唑-2,3-二醇(6c-2): 白色固体 $100 \mathrm{mg}$, 产率 64\%. m.p. 206.1 207.6 ${ }^{\circ} \mathrm{C} ;[\alpha]_{\mathrm{D}}^{25}-14.0$ (c 0.1 , $\left.\mathrm{CH}_{3} \mathrm{OH}\right) ;{ }^{1} \mathrm{H}$ NMR (400 MHz, $\left.\mathrm{CD}_{3} \mathrm{OD}\right) \delta: 7.88(\mathrm{~s}, 1 \mathrm{H})$, $7.75(\mathrm{~s}, 1 \mathrm{H}), 4.97(\mathrm{~d}, J=5.6 \mathrm{~Hz}, 1 \mathrm{H}), 4.88(\mathrm{t}, J=6.4 \mathrm{~Hz}$, $1 \mathrm{H}), 4.68$ (t, $J=7.2 \mathrm{~Hz}, 1 \mathrm{H}), 4.11$ (dd, $J=12.0,2.4 \mathrm{~Hz}$, $1 \mathrm{H}), 3.94(\mathrm{dd}, J=12.0 \mathrm{~Hz}, 5.2 \mathrm{~Hz}, 1 \mathrm{H}) ;{ }^{3} \mathrm{C}$ NMR $(100$ $\left.\mathrm{MHz}, \mathrm{CD}_{3} \mathrm{OD}\right) \delta: 161.4,145.7,131.0,126.2,126.0,119.9$, 113.2, 73.5, 65.7, 61.4, 58.9; HRMS (ESI) calcd for $\mathrm{C}_{11} \mathrm{H}_{10} \mathrm{Cl}_{2} \mathrm{~N}_{2} \mathrm{O}_{3} \mathrm{Na}[\mathrm{M}+\mathrm{Na}]^{+}$310.9966, found 310.9972 .

\section{2 .2 化合物 $7 \mathbf{a} \sim 7 \mathbf{c}$ 的合成}

以 2-脱氧- $D$-核糖为起始原料，经由上述合成 5a-1 的步骤, 得到相应的茮基保护的苯并咪唑并氮杂糖. 称 取该化合物 $(0.22 \mathrm{~g}, 0.57 \mathrm{mmol})$ 于氢气反应釜中, 加入钯 碳(0.5 equiv.)、10 mL 无水甲醇和 $3.3 \mathrm{~mL}$ 的 $1 \mathrm{~mol} / \mathrm{L}$ 盐 酸, 在氢气(2000 kPa)下室温搅拌 $40 \mathrm{~h}$. 经 TLC 检测原 料反应完全. 加入饱和碳酸氢钠溶液中和反应液至 $\mathrm{pH}$ 约等于 7. 减压蒸去溶剂, 加入适量甲醇, 析出固体盐 并过滤. 滤液浓缩后用 $200 \sim 300$ 目硅胶柱层析分离(石 油醚/乙酸乙酯, $V: V=5: 1$ )得到 $64 \mathrm{mg}$ 的产物 7a-1.

$(2 R, 3 S)-1,2,3,4$-四氢苯并 $[4,5]$ 味唑并 $[1,2-a]$ 吡啶2,3-二醇(7a-1): 白色固体，产率 55\%. m.p. 218.6 $220.1{ }^{\circ} \mathrm{C} ;[\alpha]_{\mathrm{D}}^{25}+5.0$ (c $\left.0.1, \mathrm{CH}_{3} \mathrm{OH}\right) ;{ }^{1} \mathrm{H}$ NMR $(600$ MHz, DMSO- $\left.d_{6}\right) \delta: 7.52(\mathrm{~d}, J=6.6 \mathrm{~Hz}, 1 \mathrm{H}), 7.44(\mathrm{~d}, J=$ $6.6 \mathrm{~Hz}, 1 \mathrm{H}), 7.18 \sim 7.14(\mathrm{~m}, 2 \mathrm{H}), 5.55(\mathrm{~s}, 2 \mathrm{H}), 4.20(\mathrm{~d}, J=$ $4.2 \mathrm{~Hz}, 1 \mathrm{H}), 4.14(\mathrm{dd}, J=12.0 \mathrm{~Hz}, 4.2 \mathrm{~Hz}, 2 \mathrm{H}), 4.00$ (q, $J=6.0 \mathrm{~Hz}, 1 \mathrm{H}), 3.13 \sim 3.01(\mathrm{~m}, 2 \mathrm{H}) ;{ }^{13} \mathrm{C} \mathrm{NMR}(100 \mathrm{MHz}$, DMSO- $\left.d_{6}\right) \delta: 150.9,143.7,134.9,121.9,121.7,118.5$, 109.9, 67.1, 66.9, 45.9, 30.8; HRMS (ESI) calcd for $\mathrm{C}_{11} \mathrm{H}_{12} \mathrm{~N}_{2} \mathrm{O}_{2} \mathrm{Na}[\mathrm{M}+\mathrm{Na}]^{+}$227.0796, found 227.0793. 
(1S,2S)-1-(羟甲基)-2,3-二氢- $1 H$-苯并 $[d]$ 吡咯并 [1,2- $a$ ] 咪唑-2-醇(7a-2): 白色固体 $67 \mathrm{mg}$, 产率 $58 \%$. m.p. $139.2 \sim 140.8{ }^{\circ} \mathrm{C} ;[\alpha]_{\mathrm{D}}^{25}+5.0\left(\right.$ c $\left.0.1, \mathrm{CH}_{3} \mathrm{OH}\right) ;{ }^{1} \mathrm{H}$ NMR $\left(600 \mathrm{MHz}, \mathrm{CD}_{3} \mathrm{OD}\right) \delta: 7.58 \sim 7.56(\mathrm{~m}, 1 \mathrm{H}), 7.53 \sim$ $7.51(\mathrm{~m}, 1 \mathrm{H}), 7.16(\mathrm{t}, J=4.2 \mathrm{~Hz}, 2 \mathrm{H}), 5.08(\mathrm{q}, J=6.6 \mathrm{~Hz}$, $1 \mathrm{H}), 4.55 \sim 4.53(\mathrm{~m}, 1 \mathrm{H}), 4.13(\mathrm{dd}, J=12.0,3.6 \mathrm{~Hz}, 1 \mathrm{H})$, $4.02(\mathrm{dd}, J=12.0 \mathrm{~Hz}, 5.4 \mathrm{~Hz}, 1 \mathrm{H}), 3.27$ (dd, $J=15.6 \mathrm{~Hz}$, $7.8 \mathrm{~Hz}, 1 \mathrm{H}), 2.98$ (dd, $J=16.8 \mathrm{~Hz}, 6.0 \mathrm{~Hz}, 1 \mathrm{H}) ;{ }^{13} \mathrm{C} \mathrm{NMR}$ (150 MHz, $\left.\mathrm{CD}_{3} \mathrm{OD}\right) \delta: 159.7,148.0,133.6,123.3,123.1$, 119.4, 112.3, 74.6, 63.6, 61.0, 34.3; HRMS (ESI) calcd for $\mathrm{C}_{11} \mathrm{H}_{12} \mathrm{~N}_{2} \mathrm{O}_{2} \mathrm{Na}[\mathrm{M}+\mathrm{Na}]^{+}$227.0796, found 227.0795.

(2R,3S)-7,8-二甲基- $1,2,3,4$-四氢苯并 [4,5]咪唑并 [1,2- $a$ 吡啶-2,3-二醇(7b-1): 白色固体 $66 \mathrm{mg}$, 产率 50\%. m.p. $220.5 \sim 222.1{ }^{\circ} \mathrm{C},[\alpha]_{\mathrm{D}}^{25}+30.0\left(c 0.1, \mathrm{CH}_{3} \mathrm{OH}\right) ;{ }^{1} \mathrm{H}$ NMR (400 MHz, DMSO- $\left.d_{6}\right) \delta: 7.27(\mathrm{~s}, 1 \mathrm{H}), 7.20(\mathrm{~s}, 1 \mathrm{H})$, $4.17 \sim 4.14(\mathrm{~m}, 1 \mathrm{H}), 4.11 \sim 4.07(\mathrm{~m}, 2 \mathrm{H}), 3.92(\mathrm{q}, J=6.0$ $\mathrm{Hz}, 1 \mathrm{H}), 3.51 \sim 3.53(\mathrm{~m}, 2 \mathrm{H}), 3.03(\mathrm{dd}, J=16.8 \mathrm{~Hz}, 4.8$ $\mathrm{Hz}, 1 \mathrm{H}), 2.96$ (dd, $J=16.8 \mathrm{~Hz}, 6.8 \mathrm{~Hz}, 1 \mathrm{H}), 2.31$ (s, 3H), $2.28(\mathrm{~s}, 3 \mathrm{H}) ;{ }^{13} \mathrm{C}$ NMR (100 MHz, DMSO- $\left.d_{6}\right) \delta: 149.9$, 142.3, 133.5, 130.0, 129.8, 118.7, 110.0, 67.1, 67.0, 45.9, 30.8, 20.5, 20.4; HRMS (ESI) calcd for $\mathrm{C}_{13} \mathrm{H}_{16} \mathrm{~N}_{2} \mathrm{O}_{2} \mathrm{Na}$ $[\mathrm{M}+\mathrm{Na}]^{+} 255.1109$, found 255.1113 .

(1S,2S)-1-(羟甲基)-6,7-二甲基-2,3-二氢- $1 H$-苯并 [d] 吡咯并 $[1,2-a]$ 咪唑-2-醇(7b-2): 白色固体 $66 \mathrm{mg}$, 产率 $50 \%$. m.p. $167.0 \sim 168.9{ }^{\circ} \mathrm{C} ;[\alpha]_{\mathrm{D}}^{25}+27.0$ (c 0.1 , $\left.\mathrm{CH}_{3} \mathrm{OH}\right),{ }^{1} \mathrm{H}$ NMR (400 MHz, DMSO- $\left.d_{6}\right) \delta: 7.39(\mathrm{~s}, 1 \mathrm{H})$, $7.28(\mathrm{~s}, 1 \mathrm{H}), 4.92(\mathrm{~d}, J=6.0 \mathrm{~Hz}, 1 \mathrm{H}), 4.39 \sim 4.38(\mathrm{~m}, 1 \mathrm{H})$, $4.22(\mathrm{~s}, 1 \mathrm{H}), 4.04 \sim 3.96(\mathrm{~m}, 2 \mathrm{H}), 3.79(\mathrm{dd}, J=11.2 \mathrm{~Hz}$, $6.8 \mathrm{~Hz}, 1 \mathrm{H}), 3.12$ (d, $J=7.2 \mathrm{~Hz}, 1 \mathrm{H}), 2.88 \sim 2.76$ (dd, $J=$ $16.0 \mathrm{~Hz}, 5.6 \mathrm{~Hz}, 1 \mathrm{H}) ;{ }^{13} \mathrm{C}$ NMR (100 MHz, DMSO- $\left.d_{6}\right) \delta$ : 157.8, 146.4, 131.5, 129.7, 129.3, 119.2, 112.1, 73.1, 62.5, 60.1, 33.5, 20.5, 20.4; HRMS (ESI) calcd for $\mathrm{C}_{13} \mathrm{H}_{16} \mathrm{~N}_{2}-$ $\mathrm{O}_{2} \mathrm{Na}[\mathrm{M}+\mathrm{Na}]^{+}$255.1109, found 255.1107.

$(2 R, 3 S)-7,8$-二氯-1,2,3,4-四氢苯并 [4,5]咪唑并 [1,2- $a$ ] 吡啶-2,3-二醇(7c-1): 白色固体 $76 \mathrm{mg}$, 产率 49\%. m.p. $187.2 \sim 188.9{ }^{\circ} \mathrm{C} ;[\alpha]_{\mathrm{D}}^{25}+112.0\left(c 0.1, \mathrm{CH}_{3} \mathrm{OH}\right) ;{ }^{1} \mathrm{H}$ NMR (400 MHz, $\left.\mathrm{CD}_{3} \mathrm{OD}\right) \delta: 7.68 \sim 7.63(\mathrm{~m}, 2 \mathrm{H}), 4.34(\mathrm{~s}$, $1 \mathrm{H}), 4.28(\mathrm{~s}, 1 \mathrm{H}), 4.13(\mathrm{~d}, J=4.0 \mathrm{~Hz}, 2 \mathrm{H}), 3.21(\mathrm{~s}, 2 \mathrm{H})$; ${ }^{13} \mathrm{C}$ NMR (100 MHz, $\left.\mathrm{CD}_{3} \mathrm{OD}\right) \delta: 153.2,141.8,118.7$, 111.0, 66.5, 66.5, 48.3, 45.6, 29.4; HRMS (ESI) calcd for $\mathrm{C}_{11} \mathrm{H}_{10} \mathrm{Cl}_{2} \mathrm{~N}_{2} \mathrm{O}_{2} \mathrm{Na}[\mathrm{M}+\mathrm{Na}]^{+}$295.0017, found 295.0025.

$(1 S, 2 S)$-6,7-二氯-1-(差圣甲基)-2,3-二氢- $1 H$-苯并 $[d]$ 吡 咯并 [1,2- $a$ ]咪唑-2-醇(7c-2): 白色固体 $85 \mathrm{mg}$, 产率 55\%. m.p. $193.0 \sim 194.5{ }^{\circ} \mathrm{C} ;[\alpha]_{\mathrm{D}}^{25}+18.0\left(c 0.1, \mathrm{CH}_{3} \mathrm{OH}\right) ;{ }^{1} \mathrm{H}$ NMR (400 MHz, DMSO- $\left.d_{6}\right) \delta$ : 7.95 (s, 1H), 7.78 (s, 1H),
4.94 (q, $J=6.0 \mathrm{~Hz}, 1 \mathrm{H}), 4.49 \sim 4.45(\mathrm{~m}, 1 \mathrm{H}), 3.98(\mathrm{dd}, J=$ $12.0,1.6 \mathrm{~Hz}, 1 \mathrm{H}$ ), 3.77 (dd, $J=11.6,7.6 \mathrm{~Hz}, 1 \mathrm{H}), 3.50$ (s, $2 \mathrm{H}), 3.22(\mathrm{dd}, J=16.4,7.2 \mathrm{~Hz}, 1 \mathrm{H}), 2.87$ (dd, $J=16.4 \mathrm{~Hz}$, $5.2 \mathrm{~Hz}, 1 \mathrm{H}) ;{ }^{13} \mathrm{C}$ NMR $\left(100 \mathrm{MHz}\right.$, DMSO- $\left.d_{6}\right) \delta: 162.0$, 147.5, 132.6, 130.1, 123.9, 120.1, 113.6, 73.0, 63.5, 60.0, 33.7; HRMS (ESI) calcd for $\mathrm{C}_{11} \mathrm{H}_{10} \mathrm{Cl}_{2} \mathrm{~N}_{2} \mathrm{O}_{2} \mathrm{Na}[\mathrm{M}+\mathrm{Na}]^{+}$ 295.0017, found 295.0014.

\subsection{3 化合物 $\mathbf{1 0}$ 和 $\mathbf{1 1}$ 的合成}

将化合物 $8(0.20 \mathrm{~g}, 0.91 \mathrm{mmol})$ 溶解于 $15 \mathrm{~mL}$ 无水丙 酮中, 冰浴下搅拌, 氮气保护. 待反应液充分冷却后, 向反应液中滴加浓硫酸 $(0.1 \mathrm{~mL})$, 并恢复至室温摚拌. 反应 $3 \mathrm{~h}$ 后, 薄层色谱 $(\mathrm{TCL})$ 检测反应不完全. 延长反应 时间, 板层无变化, 停止反应. 用三乙胺中和反应液至 中性，用二氯甲烷和水对反应液进行萃取，收集浓缩有 机相. 然后 200 300 目硅胶柱层析分离(石油醚/乙酸 乙酯, $V: V=2: 1$ )得到 $99 \mathrm{mg}$ 的化合物 9 .

$(3 \mathrm{a} S, 4 R, 11 \mathrm{a} R)-2,2$ 二 二甲基 - $3 \mathrm{a}, 4,11,11 \mathrm{a}$ - 四氢苯并 $[4,5]$ 咪唑并 $[1,2-a][1,3]$ 二氧杂环戊熳并 $[4,5-d]$ 吡啶-4-醇 (9): 白色固体, 产率 $42 \%$. m.p. 109.3 110.8 ${ }^{\circ} \mathrm{C} ;{ }^{1} \mathrm{H}$ NMR $\left(400 \mathrm{MHz}, \mathrm{CDCl}_{3}\right) \delta: 7.68 \sim 7.65(\mathrm{~m}, 1 \mathrm{H}), 7.33 \sim$ $7.31(\mathrm{~m}, 1 \mathrm{H}), 7.25 \sim 7.22(\mathrm{~m}, 2 \mathrm{H}), 5.29(\mathrm{~s}, 1 \mathrm{H}), 4.98(\mathrm{~d}$, $J=4.4 \mathrm{~Hz}, 1 \mathrm{H}), 4.84(\mathrm{dd}, J=10.0,5.2 \mathrm{~Hz}, 1 \mathrm{H}), 4.52(\mathrm{dd}$, $J=12.4,5.2 \mathrm{~Hz}, 1 \mathrm{H}), 4.23(\mathrm{dd}, J=12.4,5.2 \mathrm{~Hz}, 1 \mathrm{H}), 1.55$ $(3 \mathrm{H}, \mathrm{s}), 1.31(3 \mathrm{H}, \mathrm{s}) ;{ }^{13} \mathrm{C}$ NMR $\left(100 \mathrm{MHz}, \mathrm{CDCl}_{3}\right) \delta$ : $151.6,139.2,133.9,123.6,122.9,118.1,109.7,109.2$, 72.6, 64.1, 43.7, 29.7, 26.2, 23.9; HR-ESI-MS calcd for $\mathrm{C}_{14} \mathrm{H}_{16} \mathrm{~N}_{2} \mathrm{O}_{3} \mathrm{Na}[\mathrm{M}+\mathrm{Na}]^{+}$283.1058, found 283.1053.

称取化合物 $9(0.10 \mathrm{~g}, 0.38 \mathrm{mmol})$ 于 $50 \mathrm{~mL}$ 反应瓶 中, 加入 $15 \mathrm{~mL}$ 无水二氯甲烷进行溶解, 冰浴下搅拌, 氮气保护. 反应液充分冷却后, 向反应液中加入三乙胺 ( $0.16 \mathrm{~mL}, 3.0$ equiv.), 搅拌下再加入甲磺酰氯( $\mathrm{MsCl}, 0.1$ $\mathrm{mL}, 3.0$ equiv.). 撤掉冰浴室温下搅拌 $1 \mathrm{~h}$, 经 TLC 检测 原料反应完全. 用冰水淬灭反应，二氯甲烷对反应液萃 取 $(10 \mathrm{~mL} \times 3)$. 用旋转蒸发仪浓缩有机相, 然后 200 300 目硅胶柱层析分离(石油醚/乙酸乙酯, $V: V=2: 1$ ) 得到化合物 $10(102 \mathrm{mg})$ 和少量化合物 11 .

$(3 \mathrm{a} R, 4 R, 11 \mathrm{a} R)-2,2-$ 二甲基 $-3 \mathrm{a}, 4,11,11 \mathrm{a}-$ 四氢苯并 $[4,5]$ 咪唑并 $[1,2-a][1,3]$ 二氧杂环戊熳并 $[4,5-d]$ 吡啶-4-甲 磺酸酯(10): 白色固体, 产率 $80 \% .{ }^{1} \mathrm{H}$ NMR $(400 \mathrm{MHz}$, $\left.\mathrm{CDCl}_{3}\right), \delta: 7.83 \sim 7.80(\mathrm{~m}, 1 \mathrm{H}), 7.62 \sim 7.60(\mathrm{~m}, 1 \mathrm{H})$, $7.35 \sim 7.31(\mathrm{~m}, 2 \mathrm{H}), 5.63(\mathrm{dd}, J=6.0,1.2 \mathrm{~Hz}, 1 \mathrm{H}), 5.52 \sim$ $5.49(\mathrm{~m}, 1 \mathrm{H}), 4.89 \sim 4.85(\mathrm{~m}, 1 \mathrm{H}), 4.83 \sim 4.78(\mathrm{~m}, 1 \mathrm{H})$, $4.57 \sim 4.52(\mathrm{~m}, 1 \mathrm{H}), 3.11(\mathrm{~s}, 3 \mathrm{H}), 1.51(\mathrm{~s}, 3 \mathrm{H}), 1.40(\mathrm{~s}$, $3 \mathrm{H}) ;{ }^{13} \mathrm{C}$ NMR $\left(100 \mathrm{MHz}, \mathrm{CDCl}_{3}\right) \delta: 155.6,148.8,131.5$, 123.7, 123.1, 121.0, 114.7, 111.6, 81.8, 73.9, 68.3, 58.2, 
37.6, 26.5, 25.2; HR-ESI-MS calcd for $\mathrm{C}_{15} \mathrm{H}_{18} \mathrm{~N}_{2} \mathrm{O}_{5} \mathrm{SNa}$ $[\mathrm{M}+\mathrm{Na}]^{+}$361.0834, found 361.0828 .

$(3 \mathrm{a} R, 4 R, 11 \mathrm{a} R)-4$-氯-2,2-二甲基- $3 \mathrm{a}, 4,11,11 \mathrm{a}$-四氢苯 并 $[4,5]$ 咪唑并 $[1,2-a][1,3]$ 二氧杂环戊熳并 $[4,5-d]$ 吡啶 (11): 白色固体, 产率 $<5 \% .{ }^{1} \mathrm{H} \mathrm{NMR}\left(400 \mathrm{MHz}, \mathrm{CDCl}_{3}\right)$, $\delta: 7.85 \sim 7.82(\mathrm{~m}, 1 \mathrm{H}), 7.44 \sim 7.41(\mathrm{~m}, 1 \mathrm{H}), 7.39 \sim 7.30$ $(\mathrm{m}, 2 \mathrm{H}), 5.39 \sim 5.37(\mathrm{~m}, 1 \mathrm{H}), 5.02 \sim 4.96(\mathrm{~m}, 2 \mathrm{H}), 4.63 \sim$ $4.58(\mathrm{~m}, 1 \mathrm{H}), 4.36 \sim 4.31(\mathrm{~m}, 1 \mathrm{H}), 1.36(\mathrm{~d}, J=3.2 \mathrm{~Hz}$, $3 \mathrm{H}), 0.99$ (d, $J=3.2 \mathrm{~Hz}, 3 \mathrm{H}) ;{ }^{13} \mathrm{C} \mathrm{NMR}\left(100 \mathrm{MHz}, \mathrm{CDCl}_{3}\right)$ $\delta: 147.1,142.7,134.9,123.6,122.6,120.6,110.0,109.2$, 77.9, 72.3, 49.9, 43.4, 26.1, 24.2; HR-ESI-MS calcd for $\mathrm{C}_{14} \mathrm{H}_{15} \mathrm{ClN}_{2} \mathrm{O}_{2} \mathrm{Na}[\mathrm{M}+\mathrm{Na}]^{+}$301.0720, found 301.0715.

将上述冰水淬灭反应改为加入 $10 \mathrm{~mL}$ 蒸馏水, 室温 下继续摚拌 $2 \mathrm{~h}, \mathrm{TLC}$ 检测可发现氯代产物 11 和水解产 物 9(原料) 同时存在，化合物 11 的产率可提高到 48\%.

\section{2 .4 化合物 $13 \mathrm{a}$ 和 $13 \mathrm{~b}$ 的合成}

称取化合物 $9(0.20 \mathrm{~g}, 0.76 \mathrm{mmol})$ 甲磺酰化(Ms 化) 后简单后处理得到的浓缩液(主要是化合物 $\mathbf{1 0}$ ), 与无水 碳酸钾 $(150 \mathrm{mg}, 1.5$ equiv.)放于 $50 \mathrm{~mL}$ 反应瓶中, 加入 $15 \mathrm{~mL}$ 无水四氢呋喃进行溶解, 氮气保护下室温摚拌. 向反应液中加入芐胺 $(0.5 \mathrm{~mL}, 10$ equiv. $)$, 室温反应 $10 \mathrm{~h}$. 经 TLC 检测原料反应完全. 浓缩反应液并用 200 300 目硅胶柱层析分离(石油醚/乙酸乙酯, $V: V=2: 1$ ), 得 到 $106 \mathrm{mg}$ 的化合物 12a.

(3a $S, 4 R, 11 \mathrm{a} R)-N$ - 茮基-2,2-二甲基-3a,4,11,11a-四氢 苯并 $[4,5]$ 咪唑并 $[1,2-a][1,3]$ 二氧杂环戊熳并 $[4,5-d]$ 吡啶4-胺(12a): 白色固体, 产率 40\%. ${ }^{1} \mathrm{H}$ NMR (400 MHz, $\left.\mathrm{CD}_{3} \mathrm{OD}\right) \delta: 7.70 \sim 7.68(\mathrm{~m}, 1 \mathrm{H}), 7.54 \sim 7.51(\mathrm{~m}, 1 \mathrm{H})$, $7.35 \sim 7.25(\mathrm{~m}, 7 \mathrm{H}), 7.25 \sim 7.21(\mathrm{~m}, 1 \mathrm{H}), 4.78(\mathrm{dd}, J=7.2$ $\mathrm{Hz}, 2.4 \mathrm{~Hz}, 1 \mathrm{H}), 4.58 \sim 4.47(\mathrm{~m}, 2 \mathrm{H}), 4.34 \sim 4.33(\mathrm{~m}, 1 \mathrm{H})$, $3.85 \sim 3.81(\mathrm{~m}, 1 \mathrm{H}), 3.69 \sim 3.65(\mathrm{~m}, 1 \mathrm{H}), 3.39(\mathrm{~s}, 1 \mathrm{H})$, $1.33(\mathrm{~s}, 3 \mathrm{H}), 0.91(\mathrm{~s}, 3 \mathrm{H}) ;{ }^{13} \mathrm{C} \mathrm{NMR}\left(100 \mathrm{MHz}, \mathrm{CD}_{3} \mathrm{OD}\right) \delta$ : $155.1,145.5,143.6,138.9,131.8,130.5,126.5,125.8$, 122.1, 113.0, 112.5, 80.6, 77.0, 59.8, 55.1, 47.0, 29.0, 26.7; HR-ESI-MS calcd for $\mathrm{C}_{21} \mathrm{H}_{24} \mathrm{~N}_{3} \mathrm{O}_{2}[\mathrm{M}+\mathrm{H}]^{+} 350.1868$, found 350.1874 .

称取 $12 \mathrm{a}(0.25 \mathrm{~g}, 0.72 \mathrm{mmol})$ 于 $50 \mathrm{~mL}$ 反应瓶中, 加 入 $15 \mathrm{~mL}$ 无水二氯甲烷溶解, 氮气保护下室温搅拌. 充 分搅拌后向反应液中加入三氟乙酸(TFA, $3 \mathrm{~mL}$ ), 室温 搅拌 $5 \mathrm{~h}$, 经 TLC 检测原料反应完全. 反复多次加入甲 醇并用旋转蒸发仪浓缩以除去三氟乙酸. 浓缩反应液并 用 $200 \sim 300$ 目硅胶柱层析分离(石油醚/乙酸乙酯, $V$ : $V=1: 1)$, 得到 $156 \mathrm{mg}$ 的化合物 13a.

$(2 R, 3 S, 4 R)$-4-苄胺基-1,2,3,4-四氢苯并 [4,5]咪唑并 [1,2-a]吡啶-2,3-二醇(13a): 白色固体, 产率 70\%. m.p.
$116.4 \sim 117.5{ }^{\circ} \mathrm{C} ;[\alpha]_{\mathrm{D}}^{25}-12.0\left(c 0.1, \mathrm{CH}_{3} \mathrm{OH}\right) ;{ }^{1} \mathrm{H}$ NMR $\left(400 \mathrm{MHz}, \mathrm{CD}_{3} \mathrm{OD}\right) \delta: 7.70 \sim 7.68(\mathrm{~m}, 1 \mathrm{H}), 7.53 \sim 7.48$ $(\mathrm{m}, 3 \mathrm{H}), 7.38 \sim 7.28(\mathrm{~m}, 5 \mathrm{H}), 4.59 \sim 4.57(\mathrm{~m}, 1 \mathrm{H}), 4.33$ $(\mathrm{dd}, J=12.0,4.4 \mathrm{~Hz}, 1 \mathrm{H}), 4.27 \sim 4.22(\mathrm{~m}, 2 \mathrm{H}), 4.20 \sim 4.15$ $(\mathrm{m}, 2 \mathrm{H}), 4.10 \sim 4.07(\mathrm{~m}, 1 \mathrm{H}) ;{ }^{13} \mathrm{C} \mathrm{NMR}(100 \mathrm{MHz}$, $\left.\mathrm{CD}_{3} \mathrm{OD}\right) \delta: 151.7,142.5,139.7,134.3,128.1,126.8,122.3$, 118.1, 109.5, 71.1, 65.7, 56.5, 51.7, 47.2; HR-ESI-MS calcd for $\mathrm{C}_{18} \mathrm{H}_{20} \mathrm{~N}_{3} \mathrm{O}_{2}[\mathrm{M}+\mathrm{H}]^{+}$310.1555, found 310.1561 .

按相同的方法，以 4-氟茮胺为起始原料，经中间体 化合物 $12 \mathrm{~b}$ 得到了相应的目标产物 $\mathbf{1 3 b}$.

(2R,3 S, 4R)-4-(4-氟苄胺基)-1,2,3,4-四氢苯并 [4,5]咪 唑并 $[1,2-a]$ 吡啶-2,3-二醇(13b): 白色固体, 两步产率 $31 \%$. m.p. $112.5 \sim 113.7{ }^{\circ} \mathrm{C} ;[\alpha]_{\mathrm{D}}^{25}-12.0$ (c 0.1 , $\left.\mathrm{CH}_{3} \mathrm{OH}\right) ;{ }^{1} \mathrm{H}$ NMR (400 MHz, DMSO- $\left.d_{6}\right) \delta: 7.67 \sim 7.64$ $(1 \mathrm{H}, \mathrm{m}), 7.54 \sim 7.49(\mathrm{~m}, 3 \mathrm{H}), 7.26 \sim 7.15(\mathrm{~m}, 4 \mathrm{H}), 4.47(\mathrm{t}$, $J=6.0 \mathrm{~Hz}, 1 \mathrm{H}), 5.52 \sim 5.49(\mathrm{~m}, 1 \mathrm{H}), 4.89 \sim 4.85(\mathrm{~m}, 1 \mathrm{H})$, $4.83 \sim 4.78(\mathrm{~m}, 1 \mathrm{H}), 4.57 \sim 4.52(\mathrm{~m}, 1 \mathrm{H}), 3.11(\mathrm{~s}, 3 \mathrm{H})$, $1.51(\mathrm{~s}, 3 \mathrm{H}), 1.40(\mathrm{~s}, 3 \mathrm{H}) ;{ }^{13} \mathrm{C}$ NMR (150 MHz, DMSO$\left.d_{6}\right) \delta: 155.6,148.8,131.5,123.7,123.1,121.0,114.7$, 111.6, 81.8, 73.9, 68.3, 58.2, 37.6, 26.5, 25.2; HR-ESI-MS calcd for $\mathrm{C}_{18} \mathrm{H}_{19} \mathrm{FN}_{3} \mathrm{O}_{2}[\mathrm{M}+\mathrm{H}]^{+}$328.1461, found 328.1462 .

\subsection{5 化合物 $17 \mathrm{a} \sim 17 \mathrm{e}$ 的合成}

称取 11 (0.20 g, $0.72 \mathrm{mmol})$ 于 $50 \mathrm{~mL}$ 反应瓶中, 加 入 $15 \mathrm{~mL}$ 无水二氯甲烷溶解, 室温下搅拌, 氮气保护. 充分搅拌后向反应液中加入三氟乙酸(TFA, $3 \mathrm{~mL})$, 室 温摚拌 $5 \mathrm{~h}$, 经 TLC 检测原料反应完全. 反复多次加入 甲醇并用旋转蒸发仪浓缩以除去三氟乙酸. 浓缩反应液 并用 $200 \sim 300$ 目硅胶柱层析分离(石油醚/乙酸乙酯, $V: V=1: 1)$, 得到 $117 \mathrm{mg}$ 的化合物 16, 收率 $68 \%$. 化 合物 16 在干燥过程中不稳定, 会向环氧过渡态 15 转化.

称取 $16(0.10 \mathrm{~g}, 0.43 \mathrm{mmol})$ 于 $50 \mathrm{~mL}$ 反应瓶中, 加 入 $12 \mathrm{~mL}$ 无水四氢呋喃进行溶解, 室温下搅拌, 氮气保 护. 充分搅拌后向反应液中加入 $\mathrm{NaOH}(50 \mathrm{mg}, 2.0$ equiv.), 室温下继续搅拌 $2 \mathrm{~h}$, 经 TLC 检测反应完全, 反 应液经 $200 \sim 300$ 目硅胶柱层析分离(石油醚/乙酸乙酯, $V: V=1: 1)$, 得到 $69 \mathrm{mg}$ 的化合物 15 .

$(1 \mathrm{a} R, 2 R, 9 \mathrm{~b} S)-1 \mathrm{a}, 2,3,9 \mathrm{~b}-$ 四氢苯并 $[4,5]$ 咪唑并 $[1,2-a]$ 氧杂环丙熳并 $[2,3-c]$ 吡啶-2-醇(15): 白色固体, 产率 $80 \%$. m.p. $135.0 \sim 136.2{ }^{\circ} \mathrm{C} ;[\alpha]_{\mathrm{D}}^{25}-35.0$ (c 0.1 , $\left.\mathrm{CH}_{3} \mathrm{OH}\right) ;{ }^{1} \mathrm{H}$ NMR (400 MHz, CD $\left.{ }_{3} \mathrm{OD}\right) \delta: 7.67$ (dd, $J=$ 8.0, $2.4 \mathrm{~Hz}, 1 \mathrm{H},), 7.51$ (dd, $J=8.0,5.2 \mathrm{~Hz}, 1 \mathrm{H}), 7.36 \sim$ $7.29(\mathrm{~m}, 2 \mathrm{H}), 4.60 \sim 4.55(\mathrm{~m}, 1 \mathrm{H}), 4.49 \sim 4.44(\mathrm{~m}, 1 \mathrm{H})$, $4.30 \sim 4.28(\mathrm{~m}, 1 \mathrm{H}), 3.98 \sim 3.96(\mathrm{~m}, 1 \mathrm{H}), 3.74 \sim 3.68(\mathrm{~m}$, 
$1 \mathrm{H}) ;{ }^{13} \mathrm{C}$ NMR (100 MHz, $\left.\mathrm{CD}_{3} \mathrm{OD}\right) \delta: 147.5,142.1,134.3$, $123.5,122.5,118.5,109.4,65.3,56.9,42.4$; HR-ESI-MS calcd for $\mathrm{C}_{11} \mathrm{H}_{10} \mathrm{~N}_{2} \mathrm{O}_{2} \mathrm{Na}[\mathrm{M}+\mathrm{Na}]^{+}$225.0640, found 225.0643 .

称取 $15(0.10 \mathrm{~g}, 0.50 \mathrm{mmol})$ 于 $50 \mathrm{~mL}$ 反应瓶中, 加 入 $12 \mathrm{~mL}$ 无水四氢呋喃进行溶解, 室温条件下加入氨水 ( $2 \mathrm{~mL}, 10$ equiv.), 搅拌 $3 \mathrm{~h}$, 经 TLC 检测原料反应完全. 用旋转蒸发仪浓缩反应液并对其进行快速 200 300 目 硅胶柱层析分离 (二氯甲烷/甲醇, $V: V=1.5: 1$ ), 得到 $71 \mathrm{mg}$ 的化合物 17a.

按相同的方法, 分别以甲胺、乙胺、丙胺、丁胺和 环己胺为原料, 硅胶柱层析分离 (二氯甲烷/甲醇, $V$ : $V=1.5: 1 \sim 3: 1$ )得到了相应的目标产物 $\mathbf{1 7} \mathbf{b} \sim \mathbf{1 7} \mathbf{e}$.

$(2 R, 3 S, 4 R)-4$-氨基-1,2,3,4-四氢苯并 [4,5]咪唑并 [1,2-a]吡啶-2,3-二醇(17a): 白色固体, 产率 $65 \%$. m.p. $187.3 \sim 188.5{ }^{\circ} \mathrm{C} ;[\alpha]_{\mathrm{D}}^{25}+38.0\left(c 0.1, \mathrm{CH}_{3} \mathrm{OH}\right) ;{ }^{1} \mathrm{H} \mathrm{NMR}$ $\left(400 \mathrm{MHz}, \mathrm{DMSO}-d_{6}\right) \delta: 7.60 \sim 7.58(\mathrm{~m}, 1 \mathrm{H}), 7.50 \sim 7.48$ (m, 1H), $7.24 \sim 7.19(\mathrm{~m}, 2 \mathrm{H}), 4.38 \sim 4.35(\mathrm{~m}, 1 \mathrm{H}), 4.18 \sim$ $4.14(\mathrm{~m}, 2 \mathrm{H}), 4.07 \sim 4.03(\mathrm{~m}, 1 \mathrm{H}), 3.82 \sim 3.80(\mathrm{~m}, 1 \mathrm{H})$, $3.52 \sim 3.37(\mathrm{~m}, 5 \mathrm{H}) ;{ }^{13} \mathrm{C}$ NMR $\left(100 \mathrm{MHz}\right.$, DMSO- $\left.d_{6}\right) \delta$ : 154.7, 143.7, 134.9, 122.0, 118.9, 110.3, 73.9, 65.9, 51.1, 46.3, 25.3; HR-ESI-MS calcd for $\mathrm{C}_{11} \mathrm{H}_{14} \mathrm{~N}_{3} \mathrm{O}_{2}[\mathrm{M}+\mathrm{H}]^{+}$ 220.1086 , found 220.1082 .

$(2 R, 3 S, 4 R)-4$-(2-氨乙基)-1,2,3,4-四氢苯并 [4,5]咪唑 并 $[1,2-a]$ 吡啶-2,3-二醇(17b): 白色固体 $89 \mathrm{mg}$, 产率 72\%. m.p. $152.3 \sim 153.8{ }^{\circ} \mathrm{C}$; $[\alpha]_{\mathrm{D}}^{25}+9.0\left(c 0.1, \mathrm{CH}_{3} \mathrm{OH}\right)$; ${ }^{1} \mathrm{H}$ NMR (400 MHz, CD $\left.3 \mathrm{OD}\right), \delta: 7.68 \sim 7.66(\mathrm{~m}, 1 \mathrm{H})$, $7.50 \sim 7.48(\mathrm{~m}, 1 \mathrm{H}), 7.32 \sim 7.28(\mathrm{~m}, 2 \mathrm{H}), 7.54(\mathrm{t}, J=5.6$ $\mathrm{Hz}, 1 \mathrm{H}), 4.32 \sim 4.28(\mathrm{~m}, 1 \mathrm{H}), 4.19 \sim 4.13(\mathrm{~m}, 3 \mathrm{H}), 3.09 \sim$ $3.03(\mathrm{~m}, 1 \mathrm{H}), 2.93 \sim 2.88(\mathrm{~m}, 1 \mathrm{H}), 1.26 \sim 1.20(\mathrm{~m}, 3 \mathrm{H}) ;{ }^{13} \mathrm{C}$ NMR (100 MHz, $\left.\mathrm{CD}_{3} \mathrm{OD}\right) \delta: 151.7,142.5,134.3,122.5$, 122.3, 118.0, 109.5, 71.0, 65.7, 56.9, 45.1, 42.2, 13.8; HR-ESI-MS calcd for $\mathrm{C}_{13} \mathrm{H}_{18} \mathrm{~N}_{3} \mathrm{O}_{2}[\mathrm{M}+\mathrm{H}]^{+}$248.1399, found 248.1397 .

$(2 R, 3 S, 4 R)$-4-(3-氨丙基)-1,2,3,4-四氢苯并[4,5]咪唑 并[1,2- $a$ 吡啶-2,3-二醇(17c): 白色固体 $104 \mathrm{mg}$, 产率 $80 \%$. m.p. 95.9 96.6 ${ }^{\circ} \mathrm{C} ;[\alpha]_{\mathrm{D}}^{25}+9.0\left(c 0.1, \mathrm{CH}_{3} \mathrm{OH}\right) ;{ }^{1} \mathrm{H}$ NMR (400 MHz, $\left.\mathrm{CD}_{3} \mathrm{OD}\right) \delta: 7.68 \sim 7.66(\mathrm{~m}, 1 \mathrm{H}), 7.49 \sim$ $7.47(\mathrm{~m}, 1 \mathrm{H}), 7.31 \sim 7.29(\mathrm{~m}, 2 \mathrm{H}), 4.56 \sim 4.53(\mathrm{~m}, 1 \mathrm{H})$, $4.32 \sim 4.28(\mathrm{~m}, 1 \mathrm{H}), 4.18(\mathrm{~s}, 2 \mathrm{H}), 4.16 \sim 4.13(\mathrm{~m}, 1 \mathrm{H})$, $3.01 \sim 2.94(\mathrm{~m}, 1 \mathrm{H}), 2.86 \sim 2.80(\mathrm{~m}, 1 \mathrm{H}), 1.66 \sim 1.59(\mathrm{~m}$, $2 \mathrm{H}), 1.04 \sim 0.99(\mathrm{~m}, 3 \mathrm{H}) ;{ }^{13} \mathrm{C} \mathrm{NMR}\left(100 \mathrm{MHz}, \mathrm{CD}_{3} \mathrm{OD}\right) \delta$ : 151.7, 142.3, 134.3, 122.5, 122.3, 118.0, 109.5, 70.9, 65.7, 57.1, 49.9, 45.1, 22.7, 10.7; HR-ESI-MS calcd for $\mathrm{C}_{14} \mathrm{H}_{20^{-}}$ $\mathrm{N}_{3} \mathrm{O}_{2}[\mathrm{M}+\mathrm{H}]^{+}$262.1555, found 262.1554 .
$(2 R, 3 S, 4 R)$-4-(4-氨丁基)-1,2,3,4-四氢苯并 [4,5]味唑 并[1,2- $a$ 吡啶-2,3-二醇(17d): 白色固体 $103 \mathrm{mg}$, 产率 $75 \%$. m.p. $127.5 \sim 129.8{ }^{\circ} \mathrm{C} ;[\alpha]_{\mathrm{D}}^{25}+13.0$ (c 0.1 , $\mathrm{CH}_{3} \mathrm{OH}$ ); ${ }^{1} \mathrm{H}$ NMR (400 MHz, DMSO- $d_{6}$ ) $\delta: 7.68 \sim 7.47$ $(3 \mathrm{H}, \mathrm{m}), 7.19 \sim 7.16(\mathrm{~m}, 2 \mathrm{H}), 5.67(\mathrm{~s}, 1 \mathrm{H}), 4.36(\mathrm{~s}, 1 \mathrm{H})$, $4.22 \sim 4.14(\mathrm{~m}, 2 \mathrm{H}), 3.98 \sim 3.78(\mathrm{~m}, 3 \mathrm{H}), 2.92 \sim 2.84(\mathrm{~m}$, $1 \mathrm{H}), 2.74 \sim 2.68(\mathrm{~m}, 1 \mathrm{H}), 1.63 \sim 1.59(\mathrm{~m}, 2 \mathrm{H}), 1.23 \sim 1.20$ $(\mathrm{m}, 2 \mathrm{H}), 0.93 \sim 0.83(\mathrm{~m}, 5 \mathrm{H}) ;{ }^{13} \mathrm{C}$ NMR $(100 \mathrm{MHz}$, DMSO- $\left.d_{6}\right) \delta: 152.9,143.6,134.8,122.1,119.0,110.4$, 71.8, 65.3, 58.1, 48.2, 45.3, 32.4, 20.5, 14.5; HR-ESI-MS calcd for $\mathrm{C}_{15} \mathrm{H}_{22} \mathrm{~N}_{3} \mathrm{O}_{2}[\mathrm{M}+\mathrm{H}]^{+}$276.1712, found 276.1718.

$(2 R, 3 S, 4 R)$-4-(环己氨基)-1,2,3,4-四氢苯并 $[4,5]$ 味唑 并 $[1,2-a]$ 吡啶-2,3-二醇 $(\mathbf{1 7 e})$ : 白色固体 $96 \mathrm{mg}$, 产率 $64 \%$. m.p. $197.2 \sim 198.6{ }^{\circ} \mathrm{C} ;[\alpha]_{\mathrm{D}}^{25}-4.0$ (c 0.1 , $\left.\mathrm{CH}_{3} \mathrm{OH}\right) ;{ }^{1} \mathrm{H}$ NMR $\left(400 \mathrm{MHz}, \mathrm{DMSO}-d_{6}\right) \delta: 7.62 \sim 7.57$ (m, $1 \mathrm{H}), 7.52 \sim 7.46(\mathrm{~m}, 1 \mathrm{H}), 7.23 \sim 7.13(\mathrm{~m}, 2 \mathrm{H}), 5.40 \sim$ $5.34(\mathrm{~m}, 2 \mathrm{H}), 4.43 \sim 4.39(\mathrm{~m}, 1 \mathrm{H}), 4.24 \sim 4.18(\mathrm{~m}, 1 \mathrm{H})$, $4.07 \sim 4.05(\mathrm{~m}, 1 \mathrm{H}), 3.93 \sim 3.86(\mathrm{~m}, 2 \mathrm{H}), 2.91 \sim 1.89(\mathrm{~m}$, $1 \mathrm{H}), 2.05 \sim 2.01(\mathrm{~m}, 1 \mathrm{H}), 1.88 \sim 1.85(\mathrm{~m}, 2 \mathrm{H}), 1.75 \sim 1.68$ $(\mathrm{m}, 2 \mathrm{H}), 1.63 \sim 1.56(\mathrm{~m}, 1 \mathrm{H}), 1.33 \sim 1.14(\mathrm{~m}, 4 \mathrm{H}), 1.12 \sim$ $1.01(\mathrm{~m}, 2 \mathrm{H}) ;{ }^{13} \mathrm{C}$ NMR (100 MHz, DMSO- $\left.d_{6}\right) \delta: 152.9$, 143.2, 134.3, 121.5, 121.4, 118.5, 109.7, 72.0, 64.4, 54.2, 53.9, 44.5, 33.3, 32.9, 25.9, 24.5, 24.4; HR-ESI-MS calcd for $\mathrm{C}_{17} \mathrm{H}_{24} \mathrm{~N}_{3} \mathrm{O}_{2}[\mathrm{M}+\mathrm{H}]^{+} 302.1868$, found 302.1871 .

\section{2 .6 化合物 $\mathbf{1 9}$ 的合成}

称取化合物 $9(0.10 \mathrm{~g}, 0.38 \mathrm{mmol})$ 于 $50 \mathrm{~mL}$ 反应瓶 中, 加入 $15 \mathrm{~mL}$ 无水二氯甲烷进行溶解, 冰浴下搅拌, 氮气保护. 反应液充分冷却后, 向反应液中加入三乙胺 ( $0.16 \mathrm{~mL}, 3.0$ equiv.), 搅拌下再加入甲磺酰氯( $\mathrm{MsCl}, 0.1$ $\mathrm{mL}, 3.0$ equiv.). 撤掉冰浴室温下搅拌 $1 \mathrm{~h}$, 经 TLC 检测 原料反应完全. 加入 $5 \mathrm{~mL}$ 甲醇, 在氮气保护条件下搅 拌 $2 \mathrm{~h}$. 经 TLC 检测甲磺酰基化产物 $\mathbf{1 0}$ 反应完全. 二氯 甲烷对反应液萃取 $(10 \mathrm{~mL} \times 3)$. 用旋转蒸发仪浓缩有机 相, 然后 200 300 目硅胶柱层析分离(二氯甲烷/甲醇, $V: V=5: 1$ )得到化合物 $\mathbf{1 8}$. 参照上述方法, 在 $30 \%$ 三 氟乙酸条件下脱除丙叉基得到 $69 \mathrm{mg}$ 的化合物 19.

$(2 R, 3 S, 4 R)$-4-甲氧基-1,2,3,4-四氢苯并 [4,5]咪唑并 [1,2- $a]$ 吡啶-2,3-二醇 (19): 白色固体，产率 78\%. m.p. $167.4 \sim 168.3{ }^{\circ} \mathrm{C} ;[\alpha]_{\mathrm{D}}^{25}-9.0\left(c 0.1, \mathrm{CH}_{3} \mathrm{OH}\right) ;{ }^{1} \mathrm{H}$ NMR $\left(400 \mathrm{MHz}, \mathrm{DMSO}-d_{6}\right) \delta: 7.64(\mathrm{~d}, J=7.6 \mathrm{~Hz}, 1 \mathrm{H}), 7.53 \sim$ $7.51(\mathrm{~m}, 1 \mathrm{H}), 7.27 \sim 7.20(\mathrm{~m}, 2 \mathrm{H}), 5.67 \sim 5.62(\mathrm{~m}, 2 \mathrm{H})$, $4.43(\mathrm{dd}, J=4.4,2.8 \mathrm{~Hz}, 1 \mathrm{H}), 4.35 \sim 4.33(\mathrm{~m}, 1 \mathrm{H}), 4.27$ (dd, $J=11.6,5.2 \mathrm{~Hz}, 1 \mathrm{H}), 4.11(\mathrm{~s}, 1 \mathrm{H}), 3.92 \sim 3.87(\mathrm{~m}$, $1 \mathrm{H}), 3.53(\mathrm{~s}, 3 \mathrm{H}) ;{ }^{13} \mathrm{C}$ NMR (100 MHz, DMSO- $\left.d_{6}\right) \delta$ : 
$149.8,143.5,134.7,122.8,122.4,119.5,110.7,76.5,70.8$, 65.1, 58.2, 44.8; HR-ESI-MS calcd for $\mathrm{C}_{12} \mathrm{H}_{14} \mathrm{~N}_{2} \mathrm{O}_{3} \mathrm{Na}$ $[\mathrm{M}+\mathrm{Na}]^{+}$257.0902, found 257.0905.

\section{2 .7 化合物 22 和 24 的合成}

以 $L$-核糖源的苯并咪唑并氮杂糖 6a-1 为原料, 按 制备化合物 10 相同的方法, 制备化合物 21, 在三氟乙 酸条件下脱除丙叉基得到化合物 22.

(3a $S, 4 S, 11 \mathrm{~b} S)-2,2$-二甲基-3a,4,5,11b-四氢苯并 [4,5] 咪坐并 $[1,2-a][1,3]$ 二氧杂环戊熳并 $[4,5-c]$ 吡啶-4-甲磺酸 酯(21): 白色固体, 两步产率 32\%. ${ }^{1} \mathrm{H}$ NMR (600 MHz, DMSO- $\left.d_{6}\right) \delta: 7.67 \sim 7.64(\mathrm{~m}, 2 \mathrm{H}), 7.32 \sim 7.25(\mathrm{~m}, 2 \mathrm{H})$, $5.51(\mathrm{~d}, J=6.6 \mathrm{~Hz}, 1 \mathrm{H}), 5.38 \sim 5.35(\mathrm{~m}, 1 \mathrm{H}), 4.94(\mathrm{dd}, J=$ $6.6 \mathrm{~Hz}, 3.0 \mathrm{HZ}, 1 \mathrm{H}), 4.55$ (dd, $J=12.6 \mathrm{~Hz}, 4.8 \mathrm{~Hz}, 1 \mathrm{H})$, $4.34 \sim 4.31(\mathrm{~m}, 1 \mathrm{H}), 3.34(\mathrm{~s}, 3 \mathrm{H}), 1.45(\mathrm{~s}, 3 \mathrm{H}), 1.24(\mathrm{~s}$, $3 \mathrm{H}) ;{ }^{13} \mathrm{C}$ NMR (125 MHz, DMSO-d $) \delta: 147.6,143.0$, 133.7, 122.7, 122.4, 119.3, 110.6, 73.2, 73.1, 69.8, 59.8, 40.8, 37.7, 26.3, 25.3; HR-ESI-MS calcd for $\mathrm{C}_{15} \mathrm{H}_{18} \mathrm{~N}_{2-}$ $\mathrm{O}_{5} \mathrm{SNa}[\mathrm{M}+\mathrm{Na}]^{+}$361.0834, found 361.0836.

$(2 S, 3 S, 4 S)-3,4$-二羟基-1,2,3,4-四氢苯并 $[4,5]$ 味唑并 [1,2- $a$ ] 吡啶-2-甲磺酸酯(22): 无色油状物 $86 \mathrm{mg}$, 产率 $76 \%$. ${ }^{1} \mathrm{H}$ NMR (400 MHz, DMSO- $\left.d_{6}\right) \delta: 7.65 \sim 7.60(\mathrm{~m}$, $2 \mathrm{H}), 7.33 \sim 7.26(\mathrm{~m}, 2 \mathrm{H}), 5.38 \sim 5.34(\mathrm{~m}, 1 \mathrm{H}), 5.01(\mathrm{~d}, J=$ $3.2 \mathrm{~Hz}, 1 \mathrm{H}), 4.57 \sim 4.52(\mathrm{~m}, 1 \mathrm{H}), 4.39$ (d, $J=1.6 \mathrm{~Hz}, 1 \mathrm{H})$, $4.16 \sim 4.11(\mathrm{~m}, 1 \mathrm{H}), 3.37(\mathrm{~s}, 3 \mathrm{H}) ;{ }^{13} \mathrm{C} \mathrm{NMR}(100 \mathrm{MHz}$, DMSO- $\left.d_{6}\right) \delta: 152.8,143.1,134.6,122.4,122.3,118.8$, 110.6, 74.9, 69.5, 66.2, 42.2, 38.0; HR-ESI-MS calcd for $\mathrm{C}_{12} \mathrm{H}_{14} \mathrm{~N}_{2} \mathrm{O}_{5} \mathrm{SNa}[\mathrm{M}+\mathrm{Na}]^{+}$321.0521, found 321.0524 .

称取化合物 $20(0.12 \mathrm{~g}, 0.46 \mathrm{mmol})$ 于 $25 \mathrm{~mL}$ 反应瓶 中, 加入无水二氯甲烷 $5 \mathrm{~mL}$ 溶解, 再加入无水吡啶 $(1.2$ equiv., $0.04 \mathrm{~mL}),-20{ }^{\circ} \mathrm{C}$ 下搅拌 $10 \mathrm{~min}$ 后加入三氟甲 磺酸酐 $\left(\mathrm{Tf}_{2} \mathrm{O}, 1.2\right.$ equiv., $\left.0.09 \mathrm{~mL}\right)$, 氮气保护下反应 $6 \mathrm{~h}$. 经 TLC 检测原料反应完全, 反应液中加入 $15 \mathrm{~mL}$ 正己 烷, 析出油状物, 以正己烷/二氯甲烷 $(V: V=3: 1)$ 进 行抽滤(产物溶于二氯甲烷, 不溶于正己烷), 滤液浓缩 后得到淡黄色油状液体 $150 \mathrm{mg}$ 化合物 23, 产率 $84 \%$. 未经提纯，直接用于下一步反应.

称取化合物 $23(0.15 \mathrm{~g}, 0.38 \mathrm{mmol})$ 于 $25 \mathrm{~mL}$ 反应瓶 中, 加入无水四氢呋喃 $4 \mathrm{~mL}$ 作溶剂, 然后加入碳酸钾 (1.5 equiv., $79 \mathrm{mg}$ ), 室温搅拌 $10 \mathrm{~min}$ 后加入正丁胺(10 equiv., $0.38 \mathrm{~mL}$ ), 升温至回流, 氮气保护下反应 $8 \mathrm{~h}$. 经 TLC 检测原料反应完全, 反应液经减压浓缩, 200 300 目硅胶柱层析分离(乙酸乙酯/石油醚, $V: V=1: 2$ )得到 $36 \mathrm{mg}$ 的化合物 24 .

$(3 \mathrm{a} R, 11 \mathrm{~b} S)-2,2$-二甲基-3a,11b-二氢苯并[4,5]咪唑并 [1,2-a][1,3]二氧杂环戊熳并 $[4,5-c]$ 吡啶 $(\mathbf{2 4})$ : 白色固体,
产率 39\%. ${ }^{1} \mathrm{H}$ NMR (400 MHz, $\left.\mathrm{CDCl}_{3}\right), \delta: 7.82 \sim 7.79(\mathrm{~m}$, $1 \mathrm{H}), 7.44 \sim 7.42(\mathrm{~m}, 1 \mathrm{H}), 7.37 \sim 7.29(\mathrm{~m}, 2 \mathrm{H}), 7.06(\mathrm{dd}$, $J=8.0 \mathrm{~Hz}, 1.2 \mathrm{~Hz}, 1 \mathrm{H}), 5.60(\mathrm{dd}, J=8.0 \mathrm{~Hz}, 3.2 \mathrm{~Hz}, 1 \mathrm{H})$, $5.42(\mathrm{~d}, 1 \mathrm{H}), 5.11 \sim 5.08(\mathrm{~m}, 1 \mathrm{H}), 1.51(\mathrm{~s}, 3 \mathrm{H}), 1.44(\mathrm{~s}$, $3 \mathrm{H}) ;{ }^{13} \mathrm{C}$ NMR $\left(100 \mathrm{MHz}, \mathrm{CDCl}_{3}\right), \delta: 146.4,143.2,131.8$, $124.3,123.4,120.9,120.5,110.8,109.8,109.1,71.8,70.1$, 27.4, 25.8; HR-ESI-MS calcd for $\mathrm{C}_{14} \mathrm{H}_{14} \mathrm{~N}_{2} \mathrm{O}_{2} \mathrm{Na}[\mathrm{M}+$ $\mathrm{Na}]^{+}$265.0953, found 265.0956.

\section{2 .8 化合物 $\mathbf{2 8 a}$ 和 $\mathbf{2 8 b}$ 的合成}

称取 Ts 化糖原料( $3.00 \mathrm{~g}, 8.7 \mathrm{mmol})$ 溶于 $30 \mathrm{~mL}$ 甲苯 中, 加入邻苯二胺(1.2 equiv., $1.10 \mathrm{~g})$ 和三氟甲磺酸钪 (0.1 equiv., $0.43 \mathrm{~g})$, 升温至 $80{ }^{\circ} \mathrm{C}$, 氮气保护下反应 15 h. 经 TLC 检测原料反应完全, 用二氯甲烷和饱和碳酸 氢钠混合溶液萃取反应液 $(30 \mathrm{~mL} \times 3)$. 有机相用无水硫 酸钠干燥, 减压过滤. 用旋转蒸发仪浓缩滤液, 200 300 目硅胶柱层析分离(乙酸乙酯/二氯甲烷, $V: V=1$ : 2)得到 $1.2 \mathrm{~g}$ 的化合物 25 , 产率 54\%. 经质谱、熔点测试, 数据与文献[22]报道一致.

于 $5 \mathrm{~mL}$ 无水 $N, N$-二甲基甲酰胺(DMF)中加入氢化 钠 $(\mathrm{NaH}, 1.5$ equiv., $23 \mathrm{mg})$, 再加入化合物 25 (0.10 g, $0.38 \mathrm{mmol}$ )和溴乙烷 $(1.5$ equiv., $0.06 \mathrm{~mL})$. 室温反应 $4 \mathrm{~h}$, 经 TLC 检测原料反应完全. 用乙酸乙酯和水混合溶液 萃取反应液 $(5 \mathrm{~mL} \times 3)$. 有机相用无水硫酸钠干燥, 减压 过滤. 用旋转蒸发仪浓缩滤液, 200 300 目硅胶柱层析 分离(乙酸乙酯/石油醚, $V: V=1: 2$ )得到 $99 \mathrm{mg}$ 的化合 物 27a. 按照相同的方法, 以溴丁烷为原料, 合成化合 物 $27 \mathbf{b}$.

$(3 \mathrm{a} R, 4 R, 11 \mathrm{~b} S)-4$-乙氧基-2,2-二甲基- $3 \mathrm{a}, 4,5,11 \mathrm{~b}$-四氢 苯并 $[4,5]$ 咪唑并 $[1,2-a][1,3]$ 二氧杂环戊熳并 $[4,5-c]$ 吡啶 (27a): 白色固体，产率 69\%. m.p. 135.2 135.6 ${ }^{\circ} \mathrm{C}$; $[\alpha]_{\mathrm{D}}^{25}-62.0\left(c 0.1, \mathrm{CH}_{3} \mathrm{OH}\right) ;{ }^{1} \mathrm{H}$ NMR $\left(400 \mathrm{MHz}, \mathrm{CDCl}_{3}\right)$ $\delta: 7.65 \sim 7.63(\mathrm{~m}, 1 \mathrm{H}), 7.58 \sim 7.54(\mathrm{~m}, 1 \mathrm{H}), 7.36 \sim 7.27$ $(\mathrm{m}, 2 \mathrm{H}), 5.45 \sim 5.42(\mathrm{~m}, 1 \mathrm{H}), 4.95 \sim 4.93(\mathrm{~m}, 1 \mathrm{H}), 4.51 \sim$ $4.49(\mathrm{~m}, 1 \mathrm{H}), 4.08 \sim 4.05(\mathrm{~m}, 2 \mathrm{H}), 3.81 \sim 3.75(\mathrm{~m}, 2 \mathrm{H})$, $1.48(\mathrm{~s}, 3 \mathrm{H}), 1.30 \sim 1.26(\mathrm{~m}, 3 \mathrm{H}), 1.17(\mathrm{~s}, 3 \mathrm{H}) ;{ }^{13} \mathrm{C} \mathrm{NMR}$ $\left(100 \mathrm{MHz}, \mathrm{CDCl}_{3}\right), \delta: 148.4,141.9,132.9,122.4,122.1$, $117.8,110.3,109.4,72.8,72.3,69.6,64.5,39.5,25.2,24.0$, 13.7; HR-ESI-MS calcd for $\mathrm{C}_{16} \mathrm{H}_{20} \mathrm{~N}_{2} \mathrm{O}_{3} \mathrm{Na}[\mathrm{M}+\mathrm{Na}]$ 311.1371, found 311.1373.

( $3 \mathrm{a} R, 4 R, 11 \mathrm{~b} S)-4$-丁氧基-2,2-二甲基- $3 \mathrm{a}, 4,5,11 \mathrm{~b}$-四氢 苯并 $[4,5]$ 咪唑并 $[1,2-a][1,3]$ 二氧杂环戊熳并 $[4,5-c]$ 吡啶 (27b): 白色固体 $98 \mathrm{mg}$, 产率 82 \%. m.p. 121.1 $121.9{ }^{\circ} \mathrm{C} ;[\alpha]_{\mathrm{D}}^{25}-103.0\left(c 0.1, \mathrm{CH}_{3} \mathrm{OH}\right) ;{ }^{1} \mathrm{H}$ NMR $(400$ $\left.\mathrm{MHz}, \mathrm{CDCl}_{3}\right) \delta: 7.78 \sim 7.75(\mathrm{~m}, 1 \mathrm{H}), 7.37 \sim 7.26(\mathrm{~m}, 3 \mathrm{H})$, $5.44(\mathrm{~d}, J=6.4 \mathrm{~Hz}, 1 \mathrm{H}), 4.88(\mathrm{dd}, J=6.0,2.0 \mathrm{~Hz}, 1 \mathrm{H})$, 
$4.34 \sim 4.29(\mathrm{~m}, 1 \mathrm{H}), 4.14(\mathrm{t}, J=10.4 \mathrm{~Hz}, 1 \mathrm{H}), 3.88 \sim 3.84$ $(\mathrm{m}, 1 \mathrm{H}), 3.74 \sim 3.65(\mathrm{~m}, 2 \mathrm{H}), 1.70 \sim 1.63(\mathrm{~s}, 2 \mathrm{H}), 1.49(\mathrm{~s}$, $3 \mathrm{H}), 1.47 \sim 1.39(\mathrm{~m}, 2 \mathrm{H}), 1.22(\mathrm{~s}, 3 \mathrm{H}), 0.94(\mathrm{t}, J=7.6 \mathrm{~Hz}$, $3 \mathrm{H}) ;{ }^{13} \mathrm{C}$ NMR (100 MHz, $\left.\mathrm{CDCl}_{3}\right), \delta: 148.6,143.7,133.8$, $123.1,122.8,120.3,111.5,109.5,74.0,73.4,70.6,70.5$, 40.3, 31.8, 27.0, 25.7, 19.2, 13.9; HR-ESI-MS calcd for $\mathrm{C}_{18} \mathrm{H}_{24} \mathrm{~N}_{2} \mathrm{O}_{3} \mathrm{Na}[\mathrm{M}+\mathrm{Na}]^{+}$339.1684, found 339.1688.

以化合物 27 为原料，按制备化合物 13 相同的方法, 酸性条件下脱除丙叉基合成化合物 28a 和 $28 b$.

$(2 R, 3 S, 4 S)-2$-乙氧基-1,2,3,4-四氢苯并 $[4,5]$ 咪唑并 [1,2- $a$ ] 吡啶-3,4-二醇(28a): 白色固体 $143 \mathrm{mg}$, 产率 $80 \%$. m.p. $185.3 \sim 186.7{ }^{\circ} \mathrm{C} ;[\alpha]_{\mathrm{D}}^{25}-9.0\left(c \quad 0.1, \mathrm{CH}_{3} \mathrm{OH}\right) ;{ }^{1} \mathrm{H}$ NMR $\left(400 \mathrm{MHz}, \mathrm{CD}_{3} \mathrm{OD}\right) \delta: 7.60 \sim 7.58(\mathrm{~m}, 1 \mathrm{H}), 7.45 \sim$ $7.42(\mathrm{~m}, 1 \mathrm{H}), 7.25 \sim 7.22(\mathrm{~m}, 2 \mathrm{H}), 4.41(\mathrm{dd}, J=3.2,1.2$ $\mathrm{HZ}, 1 \mathrm{H}), 4.34 \sim 4.30(\mathrm{~m}, 1 \mathrm{H}), 4.06 \sim 3.97(\mathrm{~m}, 2 \mathrm{H}), 3.78 \sim$ $3.66(\mathrm{~m}, 2 \mathrm{H}), 3.56(\mathrm{~s}, 1 \mathrm{H}), 1.25(\mathrm{t}, J=6.8 \mathrm{~Hz}, 3 \mathrm{H}) ;{ }^{13} \mathrm{C}$ NMR (100 MHz, $\left.\mathrm{CD}_{3} \mathrm{OD}\right) \delta: 152.0,141.8,133.5,121.9$, 121.7, 117.5, 109.1, 73.4, 68.2, 65.6, 64.2, 62.3, 41.2, 13.7; HR-ESI-MS calcd for $\mathrm{C}_{13} \mathrm{H}_{16} \mathrm{~N}_{2} \mathrm{O}_{3} \mathrm{Na}\left[\mathrm{M}+\mathrm{Na}{ }^{+}\right.$ 271.1058 , found 271.1062 .

$(2 R, 3 S, 4 S)$-2-丁氧基-1,2,3,4-四氢苯并 [4,5]咪唑并 [1,2- $a$ ]吡啶-3,4-二醇 $(\mathbf{2 8 b})$ : 白色固体 $134 \mathrm{mg}$, 收率 75\%. m.p. $170.3 \sim 171.2{ }^{\circ} \mathrm{C} ;[\alpha]_{\mathrm{D}}^{25}-7.0\left(c 0.1, \mathrm{CH}_{3} \mathrm{OH}\right) ;{ }^{1} \mathrm{H}$ NMR (400 MHz, $\left.\mathrm{CD}_{3} \mathrm{OD}\right), \delta: 7.64 \sim 7.62(\mathrm{~m}, 1 \mathrm{H}), 7.47 \sim$ $7.45(\mathrm{~m}, 1 \mathrm{H}), 7.31 \sim 7.24(\mathrm{~m}, 2 \mathrm{H}), 4.44(\mathrm{dd}, J=3.2,1.2$ $\mathrm{Hz}, 1 \mathrm{H}), 4.34 \sim 4.31(\mathrm{~m}, 1 \mathrm{H}), 4.08 \sim 4.00(\mathrm{~m}, 2 \mathrm{H}), 3.77 \sim$ $3.71(\mathrm{~m}, 1 \mathrm{H}), 3.68 \sim 3.63(\mathrm{~m}, 1 \mathrm{H}), 1.68 \sim 1.61(\mathrm{~m}, 2 \mathrm{H})$, $1.50 \sim 1.41(\mathrm{~m}, 2 \mathrm{H}), 1.30 \sim 1.28(\mathrm{~m}, 1 \mathrm{H}), 0.97(\mathrm{t}, J=7.2$ $\mathrm{Hz}, 3 \mathrm{H}) ;{ }^{13} \mathrm{C} \mathrm{NMR}\left(100 \mathrm{MHz}, \mathrm{CD}_{3} \mathrm{OD}\right) \delta: 154.0,143.6$, $135.5,123.9,123.7,119.4,111.1,75.6,70.6,70.2,67.6$, 64.3, 43.2, 33.1, 20.3, 14.3; HR-ESI-MS calcd for $\mathrm{C}_{15} \mathrm{H}_{20} \mathrm{~N}_{2} \mathrm{O}_{3} \mathrm{Na}[\mathrm{M}+\mathrm{Na}]^{+}$299.1371, found 299.1375.

以化合物 25 为原料, 按制备化合物 22 相同的方法, 合成化合物 26.

$(2 R, 3 S, 4 S)-3,4$-二羟基-1,2,3,4-四氢苯并 $[4,5]$ 咪唑并 [1,2- $a]$ 吡啶-2-甲磺酸酯(26): 白色固体, 两步产率 58\%. m.p. $156.4 \sim 157.2{ }^{\circ} \mathrm{C} ;{ }^{1} \mathrm{H}$ NMR (400 MHz, DMSO-d $\left.d_{6}\right) \delta$ : $7.62 \sim 7.60(\mathrm{~m}, 1 \mathrm{H}), 7.56 \sim 7.54(\mathrm{~m}, 1 \mathrm{H}), 7.25 \sim 7.22(\mathrm{~m}$, $2 \mathrm{H}), 5.36 \sim 5.32(\mathrm{~m}, 1 \mathrm{H}), 4.94(\mathrm{~s}, 1 \mathrm{H}), 4.52 \sim 4.48(\mathrm{~m}$, $1 \mathrm{H}), 4.36(\mathrm{~s}, 1 \mathrm{H}), 4.13 \sim 4.00(\mathrm{~m}, 1 \mathrm{H}), 3.36(\mathrm{~s}, 3 \mathrm{H}) ;{ }^{13} \mathrm{C}$ NMR (100 MHz, DMSO- $\left.d_{6}\right) \delta$ : 153.0, 143.2, 134.5, 122.7, $122.5,119.1,110.7,75.3,69.9,66.0,42.2,38.2 ; \mathrm{C}_{12} \mathrm{H}_{14} \mathrm{~N}_{2-}$ $\mathrm{O}_{5} \mathrm{SNa}[\mathrm{M}+\mathrm{Na}]^{+}$321.0521, found 321.0527 .

\subsection{9 糖苷酶抑制活性测试}

以 $\alpha$-葡萄糖糖苷酶活性测试为例: 实验在 96 孔板
中进行, 设空白组和样品组, 每组 3 个平行. 于 96 孔板 中加入 $10 \mu \mathrm{L}$ 酶溶液 $(50 \mathrm{U} / \mathrm{mL}) 、 20 \mu \mathrm{L}$ 抑制剂 $(6 \mathrm{mmol} / \mathrm{L})$ 和 $20 \mu \mathrm{L}$ 柠檬酸-磷酸缓冲液 $(\mathrm{CPBs}, \mathrm{pH}=5.5)$, 混匀后 $37{ }^{\circ} \mathrm{C}$ 下保温 $10 \mathrm{~min}$, 加入 $50 \mu \mathrm{L}$ 对硝基苯酚 $-\alpha$-葡萄糖 苷(PNPG, $2 \mathrm{mmol} / \mathrm{L})$. 混合溶液在 $37{ }^{\circ} \mathrm{C}$ 培育 $20 \mathrm{~min}$. 以 $100 \mu \mathrm{L}$ 碳酸钠 $(1 \mathrm{~mol} / \mathrm{L})$ 淬灭反应 $10 \mathrm{~min}$. 在酶标仪波长 $405 \mathrm{~nm}$ 测定吸光度. 代入公式: 抑制率 $(\%)=\left(A_{\text {contro } L}-\right.$ $\left.A_{\text {treated }}\right) / A_{\text {control }} \times 100 \%$ 计算抑制率.

辅助材料(Supporting Information) 化合物 $6 \mathbf{6} \sim 6 \mathrm{c}$ 、 $7 a \sim 7 c 、 13 a 、 13 b 、 15 、 17 a \sim 17 e 、 19 、 26 、 28 a$ 和 $28 b$ 的核磁共振氢谱和碳谱，化合物 6a-1、6c-2、7a-1、13a、 $17 \mathrm{~b} 、 17 \mathrm{e}$ 和 19 的二维核磁谱图. 这些材料可以免费从 本刊网站(http://sioc-journal.cn/)上下载.

\section{References}

[1] Schmaltz, R. M.; Hanson, S. R.; Wong, C. H. Chem. Rev. 2011, $111,4259$.

[2] Takahashi, M.; Kizuka, Y.; Ohtsubo, K.; Gu, J. G.; Taniguchi, N. Mol. Aspects Med. 2016, 51, 56.

[3] Dai, Y. W.; Hartke, R.; Li, Chao.; Yang, Q.; Liu, J. O.; Wang, L. X. ACS Chem. Biol. 2020, 15, 2662.

[4] He, X. P.; Zeng, Y. L.; Zang, Y.; Li, J.; Field, R. A.; Chen, G. R. Carbohydr. Res. 2016, 429, 1.

[5] Wei, M. M.; Wang, Y. S.; Ye, X. S. Med. Res. Rev. 2018, 38, 1003.

[6] Stutz, A. E. Iminosugars as Glycosidase Inhibitors, Nojirimycin and Beyond, Weinheim, Wiley-VCH, 1999.

[7] Varrot, A.; Tarling, C. A.; Macdonald, J. M.; Stick, R. V.; Zechel, D. L.; Withers, S. G.; Davies, G. J. J. Am. Chem. Soc. 2003, 125, 7496.

[8] Lillelund, V. H.; Jensen, H. H.; Liang, X. F.; Bols, M. Chem. Rev. 2002, $102,515$.

[9] Ganem, B. Acc. Chem. Res. 1996, 29, 340.

[10] Kiappes, J. L.; Hill, M. L.; Alonzi, D. S.; Miller, J. L.; Iwaki, R.; Sayce, A. C.; Caputo, A. T.; Kato, A.; Zitzmann, N. ACS Chem. Biol. 2018, 13, 60 .

[11] Li, Y. X.; Jia, Y. M.; Yu, C. Y. Prog. Chem. 2018, 35, 586 (in Chinese) (李意羡, 贾月梅, 俞初一, 化学进展, 2018, 35, 586.)

[12] Sánchez-Fernández, E. M.; García-Moreno, M. I.; Arroba, A. I.; Aguilar-Diosdado, M.; Padrón, J. M.; García-Hernández, R.; Gamarro, F.; Fustero, S.; Sánchez-Aparicio, J.; Masgrau, L.; García-Fernández, J. M.; Mellet, C. O. Eur. J. Med. Chem. 2019, 182, 111604.

[13] Sánchez-Fernández, E. M.; Gonçalves-Pereira, R.; Rísquez-Cuadro, R.; Plata, G. B.; Padrón, J. M.; García-Fernández, J. M.; Mellet, C. O. Carbohydr. Res. 2016, 429, 113.

[14] Chen, H.; Hao, L.; Zhu, M.; Yang, T. Y.; Wei, S. N.; Qin, Z. B.; Zhang, P. Z.; Li, X. L. Bioorg. Med. Chem. Lett. 2014, 24, 3426.

[15] Yin, Z. Q.; Zhu, M.; Wei, S. N.; Shao, J.; Hou, Y. H.; Chen, H.; Li, X. L. Bioorg. Med. Chem. Lett. 2016, 26, 1738.

[16] Niu, L. P.; Xing, X. K.; Li, X. L.; Chem, H. Chin. J. Org. Chem. 2019, 39, 771 (in Chinese).

(牛丽萍, 邢顺凯, 李小六, 陈华, 有机化学, 2019, 39, 771.)

[17] Sun, J. J.; Kang, Y. Q.; Gao, L. G.; Lu, X.; Ju, H. H.; Li, X. L.; Chem, H. Carbohydr. Res. 2019, 478, 10.

[18] Yan, L. H.; Lui, H.; Sun, J. J.; Gao, L. G.; Lu, X.; Niu, L. P.; Li, X. 
L.; Chem, H. Carbohydr. Res. 2019, 485, 107807.

[19] Kasture, V. M.; Kalamkar, N. B.; Nair, R. J.; Joshi, R. S.; Sabharwal, S. G.; Dhavale, D. D. Carbohydr. Res. 2015, 408, 25.

[20] CCDC-2052292 (for 11) and CCDC-2059777 (for 27b) contain the supplementary crystallographic data for this paper. These data can be obtained free of charge from The Cambridge Crystallographic
Data Centre via www.ccdc.cam.ac.uk/data request/cif.

[21] Compain, P.; Martin, O. R. Iminosugars: From Synthesis to Therapeutic Applications, New York, Wiley, 2007

[22] Prasad, S. S.; Reddy, N. R.; Baskaran, S. J. Org. Chem. 2018, 83, 9604.

$(\mathrm{Lu}, \mathrm{Y}$. 